\title{
An effective guide to beyond the Standard Model physics
}

\author{
Eduard Massó \\ Grup de Física Teòrica and Institut de Física d'Altes Energies, \\ Universitat Autònoma de Barcelona, 08193 Bellaterra, Spain \\ E-mail: masso@ifae.es
}

ABSTRACT: Effective Lagrangians with dimension-six operators are widely used to analyse Higgs and other electroweak data. We show how to build a basis of operators such that each operator is linked to a coupling which is well measured or will be in the future. We choose a set of couplings such that the correspondence is one-to-one. The usual arbitrary coefficients in front of operators can be constrained quite directly using experimental data. In our framework, some important features of the Lagrangian are transparent. For example, one can clearly see the presence or absence of correlations among measurable quantities. This may be a useful guide when searching for physics beyond the Standard Model.

Keywords: Higgs Physics, Beyond Standard Model

ARXIV EPRINT: 1406.6376 


\section{Contents}

1 Introduction 2

2 The starting basis 5

2.1 CP-even basis 5

2.2 CP-odd basis 6

$\begin{array}{lll}3 & \text { The couplings } & 7\end{array}$

3.1 CP-even couplings 7

3.2 CP-odd couplings 8

3.3 From couplings to directions 9

4 The CP-even directions $\quad 9$

4.1 Higgs-only sector 9

$\begin{array}{lll}\text { 4.1.1 } a=h f f & 10\end{array}$

$\begin{array}{lll}4.1 .2 & a=h 3 & 10\end{array}$

$\begin{array}{lll}4.1 .3 & a=h(V V)_{c} & 11\end{array}$

$\begin{array}{lll}4.1 .4 \quad a=g g & 12\end{array}$

4.1.5 $a=h \gamma \gamma, h \gamma Z \quad 12$

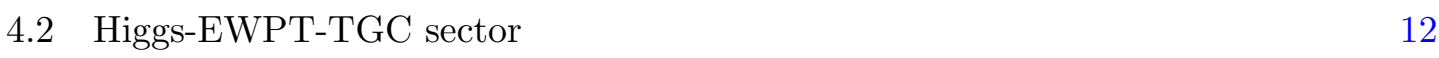

$\begin{array}{lll}4.2 .1 \quad a=g 1 Z & 13\end{array}$

$\begin{array}{lll}4.2 .2 \quad a=\kappa \gamma & 14\end{array}$

$\begin{array}{lll}4.2 .3 \quad a=Z f f & 15\end{array}$

$\begin{array}{lll}\text { 4.2.4 } & a=D V f \text { (dipoles) } & 15\end{array}$

$\begin{array}{lll}4.2 .5 & a=u R d R & 16\end{array}$

4.3 TGC-only sector 16

$\begin{array}{ll}\text { 4.3.1 } a=\lambda V & 16\end{array}$

5 The CP-odd directions $\quad 16$

$\begin{array}{llr}6 & \text { The coupling basis } & 17\end{array}$

$\begin{array}{lll}7 & \text { Discussion and conclusions } & 19\end{array}$

$\begin{array}{ll}\text { A SM Lagrangian notation } & 23\end{array}$

B Our starting basis and the basis of Grzadkowski et al. 25 


\section{Introduction}

The discovery of the Higgs boson [1, 2] at the LHC has given a significant boost to the Standard Model (SM) of particle physics: it completes the spectrum of the model and its properties are fairly consistent with the SM predictions. However, we still think that the $\mathrm{SM}$ is not the final theory of particle physics and a pressing issue is whether there will be some sign of beyond the SM (BSM) physics once the Higgs properties are known with more precision. The analysis can be done in different high-energy models, but a general tool that is now extensively used is the effective Lagrangian approach [3]-[26].

The effective Lagrangian framework allows a model independent scrutiny of electroweak data. In this paper we will adopt its linear version, suitable when the scale of BSM physics $\sim \Lambda$ is much higher than the electroweak scale (as indicated by LHC results), and the BSM degrees of freedom have been integrated out, leaving below $\Lambda$ the SM degrees of freedom, including $h$, which we assume is part of an electroweak doublet. The resulting effective theory at energies below $\Lambda$ can be described by a Lagrangian formed by gaugeinvariant operators formed by the Higgs, gauge bosons and fermion fields, in an expansion according to the dimension $d$ of the operators. The SM Lagrangian contains all $d=4$ terms. We assume that we can truncate the expansion of the effective Lagrangian at the dominant $d=6$ operators, ${ }^{1}$

$$
\mathcal{L}_{6}=\sum_{i} \frac{c_{i}}{\Lambda^{2}} \mathcal{O}_{i}
$$

The sum over $i$ runs over a basis $\left\{\mathcal{O}_{i}\right\}$ in the $d=6$ gauge-invariant operator space. One of the guiding principles to elect a particular basis should be that it simplifies the path to get to the desired goals.

To motivate our work we start with the well-know fact that the structure of the SM Lagrangian implies relations among couplings. Consider for instance the SM Lagrangian term

$$
m_{W}^{2}\left(W^{+\mu} W_{\mu}^{-}+\frac{1}{2 c_{w}^{2}} Z^{\mu} Z_{\mu}\right)\left(1+\frac{2 h}{v}+\frac{h^{2}}{v^{2}}\right)
$$

where $h$ stands for the physical Higgs boson, and $v, c_{w}=\cos \theta_{w}$, etc., are defined in appendix A. From (1.2), we see for example that the couplings $h W W$ and $h Z Z$ are related, through the expected custodial-preserving form. The couplings $h^{2} W W-h^{2} Z Z, h W W$ $h^{2} W W$, and $h Z Z-h^{2} Z Z$ are also related. These relations hold exactly at tree level, but they are slightly changed by radiative corrections. In this paper we are interested in the changes induced by $d=6$ operators. It is well-known that the modifications coming from $\mathcal{L}_{6}$ are not arbitrary. Indeed there are correlations amongst the induced modifications which stem from the fact that gauge-invariance restricts the form of the operators, and that not all the operators one can write are independent. However, as we now explain, to see clearly those correlations in complete generality may be difficult.

One uses (1.1) to predict modifications to couplings, which we denote generically by $a$. The experimental values for the different $a$ 's, obtained from measurements, constrain the $c_{i}$ 's. However, in general, a $d=6$ operator in (1.1) contributes to several couplings $a$, and

\footnotetext{
${ }^{1}$ The scale of $d=5$ operators related to $L$ non-conservation is at much higher scales.
} 
in turn each coupling $a$ gets contributions from a certain number of operators. Thus, the connection among $c_{i}$ 's and $a$ 's may be involved. When doing a numerical fit of all $c_{i}$ using observational data, one may loose sight of the correlations above mentioned and of some other general properties of the physics of effective Lagrangians. It would be desirable to have a basis where the relations among $c_{i}$ 's and $a$ 's are more direct.

The main purpose of this paper is to build a basis for $\mathcal{L}_{6}$ such that each $d=6$ operator $\mathcal{O}_{a}$ is related to a coupling $a$,

$$
\mathcal{L}_{6}=\sum_{a} \frac{c_{a}}{\Lambda^{2}} \mathcal{O}_{a}
$$

Here the sum runs over a chosen set of couplings $\{a\}$. In order that the set of gaugeinvariant operators $\left\{\mathcal{O}_{a}\right\}$ is a basis, a necessary condition is that we have as many couplings $a$ in the sum in (1.3) as operators $i$ in (1.1). We have some freedom in choosing which couplings form such a complete set; whenever it is possible we will elect the couplings that are better measured. We may say that $\left\{\mathcal{O}_{a}\right\}$ is a "coupling basis".

At the Lagrangian level, a coupling $a$ corresponds to a coefficient multiplying an operator $\widehat{\mathcal{D}}_{a}$, formed by SM fields. We say that $a$ and $\mathcal{O}_{a}$ are related when the operator $\widehat{\mathcal{D}}_{a}$ is contained in $\mathcal{O}_{a}$ when the latter is written in the unitary gauge,

$$
\frac{c_{a}}{\Lambda^{2}} \mathcal{O}_{a}^{\text {unit }}=\eta_{a}\left(\widehat{\mathcal{D}}_{a}+\delta \mathcal{D}_{a}\right)
$$

The coefficients $\eta_{a}$ are a measure of potential deviations from SM predictions (we choose the $\eta_{a}$ 's adimensional). In (1.4), $\delta \mathcal{D}_{a}$ stands for a series of coupling terms that inevitably accompany $\widehat{\mathcal{D}}_{a}$. We define

$$
\mathcal{D}_{a}=\widehat{\mathcal{D}}_{a}+\delta \mathcal{D}_{a}
$$

From a practical point of view, the Lagrangian in the unitary gauge to be added to the SM, which describes BSM effects, reads

$$
\Delta \mathcal{L}=\sum_{a} \eta_{a} \mathcal{D}_{a}
$$

This Lagrangian is written in the unitary gauge and is to be used at tree-level. Radiative corrections should be calculated with the full $\mathcal{L}_{6}$ in (1.3).

A crucial point of our work is that we manage to disentangle the couplings in $\{a\}$, i.e., $\mathcal{D}_{a}$ does not contain $\widehat{\mathcal{D}}_{b}$, for any $a$ and $b(b \neq a)$ in the set $\{a\}$ over which the sum in (1.3) extends. Then, measurement of all couplings $\left\{\eta_{a}\right\}$ determines the full Lagrangian. Given $a$ in the chosen set $\{a\}$ the expressions for $\mathcal{D}_{a}$ and $\mathcal{O}_{a}$ are unique, up to partial integrations, field redefinitions, etc. Thus, the coupling a generates a well-defined "direction" in the operator space. With a bit of language abuse we refer to $a, \mathcal{D}_{a}$ and $\mathcal{O}_{a}$ as "directions".

To reach our goals we proceed in two steps. First, we choose what we call the "starting basis" and the set of couplings $\{a\}$. The starting basis is a basis constituted by monomial operators $\mathcal{O}_{i}$ with the criterium that it is close to the $\left\{\mathcal{O}_{a}\right\}$ basis we are looking for. There is no precise definition of "close"; we simply mean that since we have some freedom in the election of $\left\{\mathcal{O}_{i}\right\}$, we will choose a basis that simplifies the algebra to be done in the second step. 
This second step consists in disentangling the starting basis $\left\{\mathcal{O}_{i}\right\}$, so that we obtain the coupling basis $\left\{\mathcal{O}_{a}\right\}$. To do it, we will work in the unitary gauge and build first the directions $\left\{\mathcal{D}_{a}\right\}$, taking care that a given $\widehat{\mathcal{D}}_{a}$ appears only in one of the directions, i.e., we have to define independent linear combinations of operators in the set $\left\{\mathcal{O}_{i}\right\}$ such that each combination contributes to one coupling of the set $\{a\}$ and not to any other. These linear combinations will give the desired $\left\{\mathcal{O}_{a}\right\}$ basis.

Once this program is done, we may say that (1.3) is the most general $d=6$ effective Lagrangian taking as directions a set of vertices that can be determined (or constrained) experimentally, with the directions independent one from the other. We could work out this program for all operators in a complete basis, which is formed by 59 operators in the case of one family ${ }^{2}$ [27]. However, we will not consider all 59 operators; we shall ignore the four-fermion operators and two operators involving only gluons. Another way to define our sub-basis is by requiring that the operators contain the Higgs field, plus two operators containing electroweak gauge bosons only. With this, we restrict our work to the sector relevant for electroweak data from Higgs physics, electroweak precision tests (EWPT) and/or triple gauge boson coupling (TGC) measurements. These restriction leaves us with 32 operators (for one family). We will use the term "basis" for them, because it is indeed a basis in the sector of experiments we consider, although they form a sub-basis of the total group of 59. In appendix B we carefully proof that our starting basis is complete in this sector.

Our results may be useful to understand some features of effective Lagrangians. Perhaps the most interesting is that it allows to see correlations among couplings in a very transparent way. For example, concerning couplings in Higgs physics, we can easily deduce which ones have discovery potential for new physics, and which ones are already constrained by EWPT tests and/or TGC measurements. For this reason we believe that the form of our effective Lagrangian may guide the search for BSM in the electroweak sector. In section 7 we discuss other potential advantages of our framework.

Let us mention the relation of our paper with other studies. Most of the work done in the field of electroweak effective Lagrangians is done using operators that are monomials. Recently, in [24], some combinations of monomials have been used, with the aim of making the connection with observables more direct, so that one can constrain the effects of effective Lagrangians in a more systematic and hierarchical way. We give a step further because we disentangle the directions completely. Actually, our work is closer in spirit to the work done in [25]. In this paper, the authors find the most general Lagrangian describing the dominant potential deviations from SM. They do it starting from the SM fields and using a variety of arguments. The construction of the Lagrangian in [25] is thus bottom-up. We build the Lagrangian using $d=6$ operators so that our approach is top-down. When we consider our Lagrangian in (1.3) in the unitary gauge and we perform a series of redefinitions of SM parameters, i.e., our (1.6), we reproduce the result in [25], as expected.

We organise the paper as follows. In section 2 we introduce the starting basis $\left\{\mathcal{O}_{i}\right\}$ and in section 3 the set of couplings $\{a\}$. Afterwards, we calculate the CP-even and CP-

\footnotetext{
${ }^{2}$ We do not consider $B$-violating operators.
} 
odd directions in section 4 and 5, respectively. We write the relations among the starting basis, the coupling basis, and the directions in section 6 . In section 7 we discuss our results, in particular some advantages of our approach are described there. We include two appendices. In appendix A we write the SM Lagrangian in order to establish some notation. In appendix B we proof the equivalence of our basis of operators and the basis in [27].

\section{The starting basis}

In this section we choose a monomial basis $\left\{\mathcal{O}_{i}\right\}$ from which we can get the coupling basis $\left\{\mathcal{O}_{a}\right\}$ in a simple way. Our election is closely related to the set of couplings which we present in the next section. For the operators we adopt the notation in [28] and [23]. Further discussion of our starting basis is done in appendix B.

We separately consider the CP-even and CP-odd cases.

\section{$2.1 \quad$ CP-even basis}

We first introduce operators which can only be tested in Higgs physics, i.e., operators such that do not lead to any physical effect when the physical Higgs field is set equal to zero. For one family, the total number of such operators is eight [23]. There are different possible choices; our election is motivated by simplicity in achieving our goals: we choose eight operators most directly related to vertices which are or will be extracted from experiment.

We have five bosonic operators

$$
\begin{array}{rlrl}
\mathcal{O}_{r} & =|\Phi|^{2}\left|D_{\mu} \Phi\right|^{2}, & \mathcal{O}_{6} & =\lambda|\Phi|^{6}, \\
\mathcal{O}_{B B} & =g^{\prime 2}|\Phi|^{2} B_{\mu \nu} B^{\mu \nu}, & \mathcal{O}_{W W} & =g^{2}|\Phi|^{2} \mathcal{W}_{\mu \nu}^{a} \mathcal{W}^{a \mu \nu} \\
\mathcal{O}_{G G} & =g_{s}^{2}|\Phi|^{2} \mathcal{G}_{\mu \nu}^{A} \mathcal{G}^{A \mu \nu} . &
\end{array}
$$

Here $\Phi$ is the complex Higgs doublet, and $\mathcal{G}_{\mu \nu}^{A}$ is the $\mathrm{SU}(3)_{c}$ gluon field strength; for other notation see appendix A. We also have three operators involving fermions

$$
\begin{array}{rlr}
\mathcal{O}_{y_{u}} & =y_{u}|\Phi|^{2} \bar{Q}_{L} \widetilde{\Phi} u_{R}, & \mathcal{O}_{y_{d}}=y_{d}|\Phi|^{2} \bar{Q}_{L} \Phi d_{R} \\
\mathcal{O}_{y_{e}} & =y_{e}|\Phi|^{2} \bar{L}_{L} \Phi e_{R}, &
\end{array}
$$

where $\widetilde{\Phi}=i \sigma_{2} \Phi^{*}$. Here and in the rest of the paper we restrict our analysis to one family.

Indeed, we see that in vacuum, $\Phi^{T}=(0, v / \sqrt{2})$, the operators in $(2.1)$ and in $(2.2)$ lead to innocuous redefinitions of SM Lagrangian parameters. We shall refer to this group of eight operators as "Higgs-only" operators.

There is a second class of operators which can be tested in $h$-physics as well as in EWPT and TGC physics. There are two bosonic operators

$$
\begin{aligned}
\mathcal{O}_{B}-\mathcal{O}_{W} & =\frac{i g^{\prime}}{2}\left(\Phi^{\dagger} \stackrel{\leftrightarrow}{D^{\mu}} \Phi\right) \partial^{\nu} B_{\mu \nu}-\frac{i g}{2}\left(\Phi^{\dagger} \sigma^{a} \stackrel{\leftrightarrow}{D^{\mu}} \Phi\right) D^{\nu} \mathcal{W}_{\mu \nu}^{a} \\
\mathcal{O}_{H B} & =i g^{\prime}\left(D^{\mu} \Phi\right)^{\dagger}\left(D^{\nu} \Phi\right) B_{\mu \nu}
\end{aligned}
$$


where $\Phi^{\dagger} \stackrel{\leftrightarrow}{D_{\mu}} \Phi \equiv \Phi^{\dagger} D_{\mu} \Phi-\left(D_{\mu} \Phi\right)^{\dagger} \Phi$. The first operator in (2.3) is the difference of two monomials, rather than a single one. This is not a problem, after all we are going to combine all these operators in the starting basis to get the coupling basis. The reason why we choose such a combination will become clear below, in eqs. (4.22) and (4.23).

In this second class, we also have fermionic operators. There are seven products of Higgs and fermion currents

$$
\begin{aligned}
\mathcal{O}_{R}^{u} & =\left(i \Phi^{\dagger} \stackrel{\leftrightarrow}{D_{\mu}} \Phi\right)\left(\bar{u}_{R} \gamma^{\mu} u_{R}\right), & \mathcal{O}_{R}^{d} & =\left(i \Phi^{\dagger} \stackrel{\leftrightarrow}{D_{\mu}} \Phi\right)\left(\bar{d}_{R} \gamma^{\mu} d_{R}\right), \\
\mathcal{O}_{R}^{e} & =\left(i \Phi^{\dagger} \stackrel{\leftrightarrow}{D_{\mu}} \Phi\right)\left(\bar{e}_{R} \gamma^{\mu} e_{R}\right), & & \\
\mathcal{O}_{L}^{q} & =\left(i \Phi^{\dagger} \stackrel{\leftrightarrow}{D_{\mu}} \Phi\right)\left(\bar{Q}_{L} \gamma^{\mu} Q_{L}\right), & \mathcal{O}_{L}^{l} & =\left(i \Phi^{\dagger} \stackrel{\leftrightarrow}{D_{\mu}} \Phi\right)\left(\bar{L}_{L} \gamma^{\mu} L_{L}\right), \\
\left.\mathcal{O}_{L}^{(3)}\right) & =\left(i \Phi^{\dagger} \sigma^{a} \stackrel{\leftrightarrow}{D_{\mu}} \Phi\right)\left(\bar{Q}_{L} \gamma^{\mu} \sigma^{a} Q_{L}\right), & \mathcal{O}_{L}^{(3) l} & =\left(i \Phi^{\dagger} \sigma^{a} \stackrel{\leftrightarrow}{D_{\mu}} \Phi\right)\left(\bar{L}_{L} \gamma^{\mu} \sigma^{a} L_{L}\right) .
\end{aligned}
$$

In addition, there are eight dipole operators

$$
\begin{array}{lll}
\mathcal{O}_{D B}^{u}=y_{u} g^{\prime}\left(\bar{Q}_{L} \sigma^{\mu \nu} u_{R} \widetilde{\Phi}\right) B_{\mu \nu}, & & \mathcal{O}_{D W}^{u}=y_{u} g\left(\bar{Q}_{L} \sigma^{\mu \nu} u_{R} \sigma^{a} \widetilde{\Phi}\right) \mathcal{W}_{\mu \nu}^{a}, \\
\mathcal{O}_{D B}^{d}=y_{d} g^{\prime}\left(\bar{Q}_{L} \sigma^{\mu \nu} d_{R} \Phi\right) B_{\mu \nu}, & & \mathcal{O}_{D W}^{d}=y_{d} g\left(\bar{Q}_{L} \sigma^{\mu \nu} d_{R} \sigma^{a} \Phi\right) \mathcal{W}_{\mu \nu}^{a}, \\
\mathcal{O}_{D B}^{e}=y_{e} g^{\prime}\left(\bar{Q}_{L} \sigma^{\mu \nu} e_{R} \Phi\right) B_{\mu \nu}, & & \mathcal{O}_{D W}^{e}=y_{e} g\left(\bar{Q}_{L} \sigma^{\mu \nu} e_{R} \sigma^{a} \Phi\right) \mathcal{W}_{\mu \nu}^{a}, \\
\mathcal{O}_{D G}^{u}=y_{u} g_{s} \bar{Q}_{L} \sigma^{\mu \nu} T^{A} u_{R} \widetilde{\Phi} \mathcal{G}_{\mu \nu}^{A}, & & \mathcal{O}_{D G}^{d}=y_{d} g_{s} \bar{Q}_{L} \sigma^{\mu \nu} T^{A} d_{R} \Phi \mathcal{G}_{\mu \nu}^{A},
\end{array}
$$

and, finally, an operator involving $u_{R}$ and $d_{R}$

$$
\mathcal{O}_{R}^{u d}=y_{u} y_{d}\left(i \widetilde{\Phi}^{\dagger} \stackrel{\leftrightarrow}{D_{\mu}} \Phi\right)\left(\bar{u}_{R} \gamma^{\mu} d_{R}\right)
$$

We refer to the whole class of 18 operators (2.3)-(2.6) as "Higgs-EWPT-TGC" operators. Some of these operators come with its hermitian conjugate.

There is a third group which actually consists of a single operator that can be only constrained using TGC measurements

$$
\mathcal{O}_{3 W}=\frac{g}{3 !} \epsilon^{a b c} \mathcal{W}_{\mu}^{a \nu} \mathcal{W}_{\nu \rho}^{b} \mathcal{W}^{c \rho \mu}
$$

Counting the operators in (2.1)-(2.7) gives a total number

$$
N_{C P+}=27
$$

of CP-even operators in the starting basis. As we said, we restrict to one fermion family.

\subsection{CP-odd basis}

We now address our interest to CP-violating operators. Actually, many operators of a complete basis, when multiplied by a complex coefficient have a CP-odd part. A classical example are the dipole operators in (2.5); if we allow for complex coefficients we have magnetic and (CP-odd) electric dipoles.

Apart from the possibility of complex coefficients, there are operators that are genuinely CP-odd. Three of them are the CP-odd siblings of $\mathcal{O}_{B B}, \mathcal{O}_{W W}, \mathcal{O}_{G G}$ in (2.1),

$$
\begin{aligned}
& \mathcal{O}_{B \widetilde{B}}=g^{\prime 2}|\Phi|^{2} B_{\mu \nu} \widetilde{B}^{\mu \nu}, \quad \mathcal{O}_{W \widetilde{W}}=g^{2}|\Phi|^{2} \mathcal{W}_{\mu \nu}^{a} \widetilde{\mathcal{W}}^{a \mu \nu}, \\
& \mathcal{O}_{G \widetilde{G}}=g_{s}^{2}|\Phi|^{2} \mathcal{G}_{\mu \nu}^{A} \widetilde{\mathcal{G}}^{A \mu \nu},
\end{aligned}
$$


with $\widetilde{V}^{\mu \nu}=\epsilon^{\mu \nu \rho \sigma} V_{\rho \sigma} / 2$. The other two are the CP-odd versions of $\mathcal{O}_{H B}$ in $(2.3)$ and $\mathcal{O}_{3 W}$ in $(2.7)$,

$$
\mathcal{O}_{H \widetilde{B}}=i g^{\prime}\left(D^{\mu} \Phi\right)^{\dagger}\left(D^{\nu} \Phi\right) \widetilde{B}_{\mu \nu}, \quad \mathcal{O}_{3 \widetilde{W}}=\frac{1}{3 !} g \epsilon^{a b c} \mathcal{W}_{\mu}^{a \nu} \mathcal{W}_{\nu \rho}^{b} \widetilde{\mathcal{W}}^{c \rho \mu}
$$

We have a total of

$$
N_{C P-}=5
$$

CP-odd operators in the starting basis.

\section{The couplings}

In this section we list the set of couplings $\{a\}$ which generate the basis $\left\{\mathcal{O}_{a}\right\}$. The set has $N_{C P+}=27$ CP-even and $N_{C P-}=5$ CP-odd couplings.

\subsection{CP-even couplings}

We start with eight couplings of the physical Higgs $h$ : to fermions (for one family), the cubic self-coupling, the custodial preserving combination of $h W W$ and $h Z Z$ (custodialviolating combinations will appear in some of the $\delta \mathcal{D}_{a}$ ), and finally to gluons, photons and $\gamma Z$,

$$
\begin{aligned}
\widehat{\mathcal{D}}_{h f f} & =h\left[\bar{f}_{L} f_{R}+\text { h.c. }\right], f=u, d, e, \quad \widehat{\mathcal{D}}_{h 3}=v h^{3}, \\
\widehat{\mathcal{D}}_{h(V V)_{c}} & =v h\left(W^{+\mu} W_{\mu}^{-}+\frac{1}{2 c_{w}^{2}} Z^{\mu} Z_{\mu}\right), \\
\widehat{\mathcal{D}}_{h g g} & =\frac{h}{v} G_{\mu \nu}^{A} G^{A \mu \nu}, \quad \widehat{\mathcal{D}}_{h \gamma \gamma}=\frac{h}{v} A^{\mu \nu} A_{\mu \nu}, \quad \widehat{\mathcal{D}}_{h \gamma Z}=\frac{h}{v} A^{\mu \nu} Z_{\mu \nu},
\end{aligned}
$$

where

$$
V_{\mu \nu}=\partial_{\mu} V_{\nu}-\partial_{\nu} V_{\mu}
$$

is the abelian part of field strength. Some of these $h$-couplings are being extracted from measurements at LHC and some will be hopefully measured in the future.

We continue listing the set $\{a\}$ with three couplings measured in processes involving TGC. We define $a=g 1 Z, \kappa \gamma$ as

$$
\begin{aligned}
\widehat{\mathcal{D}}_{g 1 Z} & =i c_{w} g\left[Z^{\mu}\left(W^{+\nu} W_{\mu \nu}^{-}-W^{-\nu} W_{\mu \nu}^{+}\right)+Z^{\mu \nu} W_{\mu}^{+} W_{\nu}^{-}\right], \\
\widehat{\mathcal{D}}_{\kappa \gamma} & =i e W_{\mu}^{+} W_{\nu}^{-}\left(A^{\mu \nu}-t_{w} Z^{\mu \nu}\right),
\end{aligned}
$$

and $a=\lambda V$ as

$$
\widehat{\mathcal{D}}_{\lambda V}=\frac{i}{m_{W}^{2}}\left(g c_{w} Z^{\mu \nu}+e A^{\mu \nu}\right) W_{\nu}^{-\rho} W_{\rho \mu}^{+} .
$$

These couplings appear in the general expression for the CP-even TGC vertex [30]. Restricting to terms generated by $d=6$ operators, we have

$$
\begin{aligned}
\delta \mathcal{L}_{3 V, C P+}= & i c_{w} g\left[\delta g_{1}^{Z} Z^{\mu}\left(W^{+\nu} W_{\mu \nu}^{-}-W^{-\nu} W_{\mu \nu}^{+}\right)\right] \\
& +i g \sum_{V=\gamma, Z} c_{V}\left[\delta \kappa_{V} V^{\mu \nu} W_{\mu}^{+} W_{\nu}^{-}+\frac{\lambda_{V}}{m_{W}^{2}} V^{\mu \nu} W_{\nu}^{-\rho} W_{\rho \mu}^{+}\right]
\end{aligned}
$$


where $c_{\gamma}=s_{w}, c_{Z}=c_{w}$, and we follow the notation shown in (3.2) for the photon $A$, the $Z$-boson and the $W^{ \pm}$-bosons.

The Lagrangian $\mathcal{L}_{6}$ implies some relations among the TGC parameters in (3.5), coming from custodial invariance preserved by the relevant $d=6$ operators and electric charge conservation [31]

$$
\delta \kappa_{Z}=\delta g_{1}^{Z}-t_{w}^{2} \delta \kappa_{\gamma}, \quad \lambda_{Z}=\lambda_{\gamma} .
$$

The five parameters in (3.5) together with the two equations (3.6) give three independent parameters. We take them to be $\delta g_{1}^{Z}, \delta \kappa_{\gamma}$ and $\lambda_{V}$ in the set $\{a\}$. The first operator in (3.3) corresponds to $\delta g_{1}^{Z}=\delta \kappa_{Z}$ and the second to $\delta \kappa_{\gamma}=t_{w}^{-2} \delta \kappa_{Z}$, while other couplings are set equal to zero. The relation $\lambda_{Z}=\lambda_{\gamma}$ in (3.6) leads us to define the coupling the way we do it in (3.4). The deviation of the TGC couplings from the SM prediction was constrained by LEP-2, and is supposed to be further investigated by LHC.

We continue with seven couplings that are very well measured at the $Z$-pole,

$$
\widehat{\mathcal{D}}_{Z e R}=Z_{\mu} \bar{e}_{R} \gamma^{\mu} e_{R}, \quad \widehat{\mathcal{D}}_{Z u R}=Z_{\mu} \bar{u}_{R} \gamma^{\mu} u_{R}, \quad \widehat{\mathcal{D}}_{Z d R}=Z_{\mu} \bar{d}_{R} \gamma^{\mu} d_{R},
$$

and

$$
\begin{array}{ll}
\widehat{\mathcal{D}}_{Z e L}=Z_{\mu} \bar{e}_{L} \gamma^{\mu} e_{L}, & \widehat{\mathcal{D}}_{Z u L}=Z_{\mu} \bar{u}_{L} \gamma^{\mu} u_{L}, \\
\widehat{\mathcal{D}}_{Z d L}=Z_{\mu} \bar{d}_{L} \gamma^{\mu} d_{L}, & \widehat{\mathcal{D}}_{Z \nu L}=Z_{\mu} \bar{\nu}_{L} \gamma^{\mu} \nu_{L} .
\end{array}
$$

These seven couplings are tightly bounded from $Z$-pole measurements. We do not use the $S$ parameter [29] because it would be redundant. Indeed, $S$ is equivalent to a universal part of the seven couplings (3.7) and (3.8).

In our list there also are eight dipole couplings, defined as

$$
\widehat{\mathcal{D}}_{D G q}=\frac{1}{v} \bar{q}_{L} \sigma^{\mu \nu} T^{A} q_{R} G_{\mu \nu}^{A}+\text { h.c. }, \quad \widehat{\mathcal{D}}_{D V f}=\frac{1}{v} \bar{f}_{L} \sigma^{\mu \nu} f_{R} V_{\mu \nu}+\text { h.c. },
$$

with $q=u, d$ and $V=A, Z, f=u, d$,e. See ref. [26] for updated constraints on dipole couplings. Finally, we also include

$$
\widehat{\mathcal{D}}_{u R d R}=\bar{u}_{R} \gamma^{\mu} d_{R} W_{\mu}^{+}+\text {h.c. . }
$$

The total number of couplings in eqs. (3.1), (3.3), (3.4), and (3.7)-(3.10) is $N_{C P+}=27$.

\section{$3.2 \quad$ CP-odd couplings}

In the list of CP-odd couplings we have the three vertices $h$-gluon-gluon, $h$-photon-photon, and $h$-photon- $Z$,

$$
\widehat{\mathcal{D}}_{h g \widetilde{g}}=\frac{h}{v} G_{\mu \nu}^{A} \widetilde{G}^{A \mu \nu}, \quad \widehat{\mathcal{D}}_{h \gamma \widetilde{\gamma}}=\frac{h}{v} A_{\mu \nu} \widetilde{A}^{\mu \nu}, \quad \widehat{\mathcal{D}}_{h \gamma \widetilde{Z}}=\frac{h}{v} A_{\mu \nu} \widetilde{Z}^{\mu \nu},
$$

and two more appearing in the expression for the CP-odd TGC vertex [30]

$$
\delta \mathcal{L}_{3 V, C P-}=i g \sum_{V=\gamma, Z} c_{V}\left[\delta \widetilde{\kappa}_{V} \widetilde{V}^{\mu \nu} W_{\mu}^{+} W_{\nu}^{-}+\frac{\widetilde{\lambda}_{V}}{m_{W}^{2}} \widetilde{V}^{\mu \nu} W_{\nu}^{-\rho} W_{\rho \mu}^{+}\right]
$$


where $c_{\gamma}=s_{w}, c_{Z}=c_{w}$. We have restricted to parameters induced by $d=6$ operators. In addition, one has that

$$
\delta \widetilde{\kappa}_{Z}=-t_{w}^{2} \delta \widetilde{\kappa}_{\gamma}, \quad \widetilde{\lambda}_{\gamma}=\widetilde{\lambda}_{Z}
$$

We take as couplings in the set $\{a\}$ the following two:

$$
\begin{aligned}
& \widehat{\mathcal{D}}_{\widetilde{\kappa \gamma}}=i e W_{\mu}^{+} W_{\nu}^{-}\left(\widetilde{A}^{\mu \nu}-t_{w} \widetilde{Z}^{\mu \nu}\right) \\
& \widehat{\mathcal{D}}_{\widetilde{\lambda V}}=\frac{i}{M_{W}^{2}}\left(g c_{w} \widetilde{Z}^{\mu \nu}+e \widetilde{A}^{\mu \nu}\right) W_{\nu}^{-\rho} W_{\rho \mu}^{+} .
\end{aligned}
$$

There are $N_{C P-}=5$ CP-odd couplings, as expected.

\subsection{From couplings to directions}

In the next section we build the set of directions $\left\{\mathcal{D}_{a}\right\}$. The material we have is on the one hand the starting basis operators in section 2 and on the other hand the couplings we have introduced in the present section. The attentive reader has noticed a parallelism among groups of operators in section 2 and groups of couplings in section 3. For example, the eight Higgs-only operators in (2.1) and (2.2) are related to the eight Higgs couplings in (3.1). This of course has a reason. The number has to be the same, if not we would have either a redundancy or missing elements in the basis. However, not all possibilities are allowed. It is not possible to change the elected couplings arbitrarily. For instance one would be tempted to dispose of the $h^{3}$ coupling, and to elect another one that will presumably be measured before, for example a non-custodial $h W W-h Z Z$ coupling. This is not allowed, because in any $d=6$ basis one has one operator giving essentially $h^{3}$, so that we cannot ignore it. There are allowed possibilities to choose other couplings instead of the election (3.1). However, this new election would mean to introduce couplings that are less well measured, and this is why we stick to (3.1).

Apart from the Higgs-only sector, the parallelism we have alluded is obvious in the correspondence $(2.4) \leftrightarrow(3.7)-(3.8),(2.5) \leftrightarrow(3.9),(2.6) \leftrightarrow(3.10)$, and $(2.7) \leftrightarrow(3.4)$. We will also see that there is a correspondence $(2.3) \leftrightarrow(3.3)$. Finally, for the CP-odd sector the correspondence reads $(2.9) \leftrightarrow(3.11)$, and $(2.10) \leftrightarrow(3.14)$.

\section{The CP-even directions}

In this section we calculate the directions $\mathcal{D}_{a}$, in the case that $\mathcal{L}_{6}$ respects $\mathrm{CP}$. We use the notation shown in appendix A, with $H / \sqrt{2}$ being the neutral part of the Higgs doublet, and $H=v+h$, with $h$ the physical Higgs field and $v \simeq 243 \mathrm{GeV}$ as given in (A.13).

\subsection{Higgs-only sector}

Now we use the eight operators in (2.1) and (2.2) to find the eight directions corresponding to the couplings (3.1). 


\subsection{1 $a=h f f$}

The three Higgs-fermion-fermion directions $a=h f f$ can be simply obtained from

$$
\mathcal{O}_{h f f}=\mathcal{O}_{y_{f}} \rightarrow H^{3}\left(\bar{f}_{L} f_{R}+\text { h.c. }\right) \text {, }
$$

with $f=u, d, e$. Here and in the following we denote by an arrow the expression of the $d=6$ operators in the unitary gauge, up to signs, constant factors, and/or coupling constants.

We write the factor $H^{3}$ in $(4.1)$ as

$$
H^{3}=H v^{2}+H\left(H^{2}-v^{2}\right) .
$$

In this expression the part $H v^{2}$ leads to a term proportional to the Yukawa term $y_{f} H \bar{f} f$ in the SM Lagrangian, and it can reabsorbed by redefining $y_{f}$. Expanding the part $H\left(H^{2}-v^{2}\right)$ leads to

$$
\mathcal{D}_{h f f}=\left(h P_{2}\right)\left[\bar{f}_{L} f_{R}+\text { h.c. }\right]
$$

with

$$
P_{2}=1+\frac{3 h}{2 v}+\frac{h^{2}}{2 v^{2}}
$$

Here and in the following $P_{n}, \ldots$ are order- $n$ polynomials in $h$. They all start with an independent term equal to one, so that higher $h$-powers lead to vertices with additional $h$ 's in the vertex. For example, in (4.3) we see that setting $P_{2}=1$ gives us the desired operator $\widehat{\mathcal{D}}_{h f f}=h\left(\bar{f}_{L} f_{R}+\right.$ h.c $)$, which defines the coupling. As expected, accompanying the operator $\widehat{\mathcal{D}}_{h f f}$ there are other terms $\delta \mathcal{D}_{h f f}$, see (1.4) and (1.5). One can easily generalise this result to the case of three families. To obtain $\mathcal{D}_{h f f}$ from $\mathcal{O}_{h f f}^{\text {unit }}$ we have to make an unobservable redefinition of the Yukawa couplings $y_{f}$.

\subsection{2 $a=h 3$}

For the triple Higgs vertex $h^{3}$, which hopefully will be measured in the future, we only need $\mathcal{O}_{6}$,

$$
\mathcal{O}_{h 3}=\mathcal{O}_{6} \rightarrow H^{6}
$$

which can be written as

$$
H^{6} \sim\left(H^{2}-v^{2}\right)^{3} .
$$

Indeed, when expanding the cube power in the rhs, the terms $H^{4}$ and $H^{2}$ can be absorbed in parameters of the $V(H)$ potential, see (A.12). The symbol $\sim$ will be used to relate terms in the Lagrangian that are equivalent up to redefinitions and/or partial integrations.

From (4.6) we get the expression

$$
\mathcal{D}_{h 3}=v h^{3} P_{1}^{3},
$$

with

$$
P_{1}=1+\frac{h}{2 v} .
$$

To obtain $\mathcal{D}_{h 3}$ from $\mathcal{O}_{h 3}^{\text {unit }}$ we have to make an unobservable redefinition of the $V(H)$ parameters. 


\subsection{3 $a=h(V V)_{c}$}

Next we consider the coupling of $h$ to $W W$ and $Z Z$ in (3.1). The relevant operator in the starting basis is $\mathcal{O}_{r}$. It can written as

$$
\mathcal{O}_{r} \sim\left(|\Phi|^{2}-\frac{v^{2}}{2}\right)\left|D_{\mu} \Phi\right|^{2},
$$

because the part proportional to $v^{2}$ adds to the kinetic $\Phi$-term and it can be reabsorbed by redefining $\Phi$ and bare parameters in the SM Lagrangian.

Eq. (4.9), in the unitary gauge, gives

$$
\frac{1}{4}\left(H^{2}-v^{2}\right)\left(\partial^{\mu} H \partial_{\mu} H+\frac{g^{2}}{2} H^{2} W^{+\mu} W_{\mu}^{-}+\frac{g^{2}}{4 c_{w}^{2}} H^{2} Z^{\mu} Z_{\mu}\right) .
$$

We get the desired term $h(V V)_{c}$, but to get rid of the term $\left(\partial^{\mu} H\right)^{2}$ we integrate by parts,

$$
\left(H^{2}-v^{2}\right)\left(\partial^{\mu} H \partial_{\mu} H\right) \sim-\left(v+\frac{h}{3}\right) h^{2} \partial^{\mu} \partial_{\mu} H
$$

and use the EoM in eq. (A.15) for $\partial^{\mu} \partial_{\mu} H$. The resulting expression contains a $h^{3}$-term. Since we wish the directions to be independent one from the other, we have to subtract a term proportional to (4.7). The end result for the $h(V V)_{c}$ direction is given by

$$
\mathcal{D}_{h(V V)_{c}}=v\left(h P_{3}\right)\left[W^{+\mu} W_{\mu}^{-}+\frac{1}{2 c_{w}^{2}} Z^{\mu} Z_{\mu}\right]+\frac{m_{f}}{4 m_{W}^{2}}\left(h^{2} Q_{1}\right) \bar{f} f+\frac{m_{h}^{2}}{12 m_{W}^{2}}\left(h^{4} Q_{2}\right),
$$

where there is a sum over all fermions $f$, and we have defined

$$
\begin{aligned}
& P_{3}=1+\frac{2 h}{v}+\frac{4 h^{2}}{3 v^{2}}+\frac{h^{3}}{3 v^{3}}, \\
& Q_{1}=1+\frac{h}{3 v}, \\
& Q_{2}=1+\frac{3 h}{4 v}+\frac{h^{2}}{8 v^{2}} .
\end{aligned}
$$

We notice the appearance of the custodial-preserving combination

$$
\widehat{\mathcal{D}}_{h(V V)_{c}}=v h\left(W^{+\mu} W_{\mu}^{-}+\frac{1}{2 c_{w}^{2}} Z^{\mu} Z_{\mu}\right) .
$$

as the coupling $a=h(V V)_{c}$ in the basis set $\{a\}$, as we anticipated in (3.1).

At the $d=6$ operator level, this direction is obtained from the combination of two operators in the starting basis: $\mathcal{O}_{r}$ and $\mathcal{O}_{6}$. In order that the latter cancels de cubic Higgs terms, the precise combination is

$$
\mathcal{O}_{h(V V)_{c}}=\mathcal{O}_{r}-\frac{1}{2} \mathcal{O}_{6} .
$$

To get $\mathcal{D}_{h(V V)_{c}}$ from $\mathcal{O}_{h(V V)_{c}}$, one has to set the unitary gauge and redefine SM parameters, specifically the Yukawa couplings and $V(H)$ parameters. 


\subsection{4 $a=g g$}

To determine the direction Higgs-gluon-gluon, in (3.1), we only need $\mathcal{O}_{G G}$,

$$
\mathcal{O}_{h g g}=\mathcal{O}_{G G} \rightarrow H^{2} \mathcal{G}_{\mu \nu}^{A} \mathcal{G}^{A \mu \nu} .
$$

We decompose the factor $H^{2}$ in (4.16) as

$$
H^{2}=v^{2}+\left(H^{2}-v^{2}\right) .
$$

The term $v^{2} \mathcal{G}_{\mu \nu}^{A} \mathcal{G}^{A \mu \nu}$ can be ignored because it is proportional to the kinetic term for the gluon field, and thus can be reabsorbed in the coupling $g_{s}$. Therefore,

$$
\mathcal{D}_{h g g}=\left(\frac{h}{v} P_{1}\right) \mathcal{G}_{\mu \nu}^{A} \mathcal{G}^{A \mu \nu}
$$

Notice that we get (4.18) from $\mathcal{O}_{G G}$ when redefining $g_{s}$.

\subsection{5 $a=h \gamma \gamma, h \gamma Z$}

The directions $\mathcal{O}_{h \gamma \gamma}$ and $\mathcal{O}_{h \gamma Z}$ correspond to combinations of $\mathcal{O}_{B B}$ and $\mathcal{O}_{W W}$. We have

$$
\begin{aligned}
\mathcal{O}_{B B} & \rightarrow H^{2} B_{\mu \nu} B^{\mu \nu} \sim\left(H^{2}-v^{2}\right) B_{\mu \nu} B^{\mu \nu}, \\
\mathcal{O}_{W W} & \rightarrow H^{2} \mathcal{W}_{\mu \nu}^{a} \mathcal{W}^{a \mu \nu} \sim\left(H^{2}-v^{2}\right) \mathcal{W}_{\mu \nu}^{a} \mathcal{W}^{a \mu \nu}
\end{aligned}
$$

where we obtain the two r.h.s. parts using (4.17) and reabsorbing the corresponding $v^{2}$-part in $g^{\prime}$ and $g$, as we have done above for $a=h g g$. We can form two linear combinations of the r.h.s. parts in (4.19), one which cancels the vertex $h \gamma Z$ and the other which cancels $h \gamma \gamma$. The first combination defines the direction $a=h \gamma \gamma$

$$
\mathcal{D}_{h \gamma \gamma}=\left(\frac{h}{v} P_{1}\right)\left[\mathcal{A}_{\mu \nu} \mathcal{A}^{\mu \nu}+\mathcal{Z}_{\mu \nu} \mathcal{Z}^{\mu \nu}+2 \mathcal{W}_{\mu \nu}^{+} \mathcal{W}^{-\mu \nu}\right],
$$

and the second defines the direction $a=h \gamma Z$

$$
\mathcal{D}_{h \gamma Z}=\left(\frac{h}{v} P_{1}\right)\left[\mathcal{A}_{\mu \nu} \mathcal{Z}^{\mu \nu}+\frac{c_{w}^{2}-s_{w}^{2}}{2 c_{w} s_{w}} \mathcal{Z}_{\mu \nu} \mathcal{Z}^{\mu \nu}+\frac{c_{w}}{s_{w}} \mathcal{W}_{\mu \nu}^{+} \mathcal{W}^{-\mu \nu}\right]
$$

The non-abelian parts of the field strengths $\mathcal{A}_{\mu \nu}, \mathcal{Z}_{\mu \nu}, \mathcal{W}_{\mu \nu}^{ \pm}$are shown in (A.5).

In these directions, we get vertices of the form $h V_{\mu \nu} V^{\mu \nu}, V=Z, W$. We have defined the direction $h(V V)_{c}$ in terms of couplings $h V_{\mu} V^{\mu}$. These two types of couplings can be distinguished experimentally, so that $h \gamma Z, h \gamma \gamma$ and $h(V V)_{c}$ are independent directions. The terms $h V_{\mu \nu} V^{\mu \nu}$ in (4.21) induce custodial breaking contributions to $h \rightarrow V V^{(*)}$, where $V^{(*)}$ is an off-mass-shell $Z$ or $W$. Finally, notice that get the $\mathcal{D}_{a}$ from the corresponding $\mathcal{O}_{a}^{\text {unit }}$ for $a=h \gamma \gamma, h \gamma Z$, we need to redefine the couplings $g, g^{\prime}$.

\subsection{Higgs-EWPT-TGC sector}

Let us now turn our attention to operators which induce changes in $h$-physics, EWPT, and TGC. We first discuss $\mathcal{O}_{B}-\mathcal{O}_{W}$ and $\mathcal{O}_{H B}$ in (2.3), which are related to the couplings in (3.3), and afterwards we will move to directions with fermions. 


\subsection{1 $a=g 1 Z$}

Consider $\mathcal{O}_{W}$,

$$
\mathcal{O}_{W}=\frac{i g}{2}\left(\Phi^{\dagger} \sigma^{a} \stackrel{\leftrightarrow}{D^{\mu}} \Phi\right) D^{\nu} \mathcal{W}_{\mu \nu}^{a} \rightarrow H^{2}\left(g W^{a \mu}-g^{\prime} \delta^{a 3} B^{\mu}\right) D^{\nu} \mathcal{W}_{\mu \nu}^{a}
$$

In the r.h.s. we see there is a contribution $v^{2}\left(g / c_{w}\right) Z^{\mu} g \epsilon^{3 b c} W^{b \nu} W_{\mu \nu}^{c}$, i.e., a contribution to $\delta g_{1}^{Z}$. However, there is a contribution $-v^{2} g^{\prime} B^{\mu} \partial^{\nu} W_{\mu \nu}^{3}$, i.e. a kinetic $W^{3}-B$ mixing. This contributes to the $S$-parameter [29], equivalent to an universal modification of the $Z f f$ vertices. Since we wish to keep all the $Z f f$ directions -see (3.7)- we should subtract the contribution of $\mathcal{O}_{W}$ to $S$. The simplest way to proceed is to consider the operator [28]

$$
\mathcal{O}_{B}=\frac{i g^{\prime}}{2}\left(\Phi^{\dagger} \stackrel{\leftrightarrow}{D^{\mu}} \Phi\right) \partial^{\nu} B_{\mu \nu} \rightarrow H^{2} Z^{\mu} \partial^{\nu} B_{\mu \nu}
$$

which contributes to $S$ but not to $\delta g_{1}^{Z}$. The right combination to get rid of $S$ is the difference $\mathcal{O}_{B}-\mathcal{O}_{W}[28]$. This is the reason we started in (2.3) with such a combination.

To determine the direction corresponding to $\delta g_{1}^{Z}$ we decompose the factor $H^{2}$ in (4.22) and (4.23) as in (4.17). The term with $v^{2}$, after partial integration and $g, g^{\prime}$ redefinitions contains the term $\delta g_{1}^{Z}$, accompanied by quartic gauge couplings. Concerning the term with $H^{2}-v^{2}$ we use the EoM for the gauge fields (A.16). The result is

$$
\begin{aligned}
& i c_{w} g\left[Z^{\mu}\left(W^{+\nu} \mathcal{W}_{\mu \nu}^{-}-\text {h.c. }\right)+\mathcal{Z}^{\mu \nu} W_{\mu}^{+} W_{\nu}^{-}\right] \\
& -\frac{1}{v^{2}}\left(H^{2}-v^{2}\right)\left[\frac{g^{2}-g^{\prime 2}}{4} H^{2} Z^{\mu} Z_{\mu}+\frac{g^{2} c_{w}^{2}}{2} H^{2} W^{+\mu} W_{\mu}^{-}\right. \\
& \left.+\left(g^{\prime} s_{w} J_{Y}^{\mu}+g c_{w} J^{3 \mu}\right) Z_{\mu}+\frac{c_{w}^{2} g}{\sqrt{2}}\left(J_{W}^{\mu} W_{\mu}^{+}+\text {h.c }\right)\right] .
\end{aligned}
$$

The fermionic currents are defined in (A.9) and (A.10).

Notice the appearence of the vertices $h W_{\mu} W^{\mu}$ and $h Z_{\mu} Z^{\mu}$ in (4.24). We should add to (4.24) a term proportional to (4.12) so that $\widehat{\mathcal{D}}_{h(V V)_{c}}$ as given in (4.14) cancels out, i.e., $a=h(V V)_{c}$ and $a=g 1 Z$ are independent one from each other. This will generate the presence of fermionic terms coupling to several Higgs and Higgs self-interactions in the direction $a=g 1 Z$,

$$
\begin{aligned}
\mathcal{D}_{g 1 Z}= & i g c_{w}\left[Z^{\mu}\left(W^{+\nu} \mathcal{W}_{\mu \nu}^{-}-\text {h.c. }\right)+\mathcal{Z}^{\mu \nu} W_{\mu}^{+} W_{\nu}^{-}\right] \\
& -\frac{g^{2} c_{w}^{2}}{2}\left(\frac{h}{v} S_{2}\right) \widehat{\mathcal{D}}_{h(V V)_{c}}+\frac{g^{\prime 2}}{2}\left(h Q_{3}\right) v Z^{\mu} Z_{\mu} \\
& +g^{2} c_{w}^{2}\left[\frac{m_{f}}{4 m_{W}^{2}}\left(h^{2} Q_{1}\right) \bar{f} f+\frac{m_{h}^{2}}{12 m_{W}^{2}}\left(h^{4} Q_{2}\right)\right] \\
& -2\left(\frac{h}{v} P_{1}\right)\left[\left(g \frac{c_{w}^{2}-s_{w}^{2}}{c_{w}} J_{Z}^{\mu}+2 s_{w} c_{w} e J_{e m}^{\mu}\right) Z_{\mu}+\frac{c_{w}^{2} g}{\sqrt{2}}\left(J_{W}^{\mu} W_{\mu}^{+}+\text {h.c }\right)\right] .
\end{aligned}
$$

Here we have substituted $s_{w} g^{\prime} J_{Y}+g c_{w} J^{3}=\left(g / c_{w}\right)\left(c_{w}^{2}-s_{w}^{2}\right) J_{Z}+2 s_{w} c_{w} e J_{e m}$. The polynomials $Q_{1}$ and $Q_{2}$ are given in (4.13), and we have introduced

$$
\begin{aligned}
& S_{2}=1+\frac{4 h}{3 v}+\frac{h^{2}}{3 v^{2}}, \\
& Q_{3}=1+\frac{5 h}{2 v}+\frac{2 h^{2}}{v^{2}}+\frac{h^{3}}{2 v^{3}} .
\end{aligned}
$$

We define the direction (4.25) so that the coefficient $\eta_{g 1 Z}=\delta g_{1}^{Z}=\delta \kappa_{Z}$ in (1.3). 
We have defined as one of our directions the custodial-preserving structure $\widehat{\mathcal{D}}_{h(V V)_{c}}$ in (4.14). Obviously, we should allow for the possibility of a custodial-breaking term in the Lagrangian. This can be done with any combination of $h W_{\mu} W^{\mu}$ and $h Z_{\mu} Z^{\mu}$ different from the one in (4.14). We choose $h Z_{\mu} Z^{\mu}$ as the custodial-breaking term -that is why it appears in (4.25). In our approach this is a simple way to describe custodial-symmetry breaking effects.

As we said, to obtain the operator $\mathcal{O}_{g 1 Z}$ in the coupling basis we have to combine a piece proportional to $\mathcal{O}_{h(V V)_{c}}$ in (4.15) with $\mathcal{O}_{B}-\mathcal{O}_{W}$. The exact combination is

$$
\mathcal{O}_{g 1 Z}=\mathcal{O}_{B}-\mathcal{O}_{W}-g^{2}\left(\mathcal{O}_{r}-\frac{1}{2} \mathcal{O}_{6}\right)
$$

In the unitary gauge, $\mathcal{O}_{g 1 Z}$ gives $\mathcal{D}_{g 1 Z}$ provided we make some redefinitions in the SM Lagrangian.

\subsection{2 $a=\kappa \gamma$}

Now it is the turn of $\kappa_{\gamma}$, related to $\mathcal{O}_{H B}$,

$$
\mathcal{O}_{H B} \rightarrow i g H^{2} W_{\mu}^{-} W_{\nu}^{+} B^{\mu \nu}+\frac{2}{c_{w}} H\left(\partial_{\nu} H\right) Z_{\mu} B^{\mu \nu} .
$$

Indeed, the first term in the r.h.s. contains the coupling $\widehat{\mathcal{D}}_{\kappa \gamma}$ (for $H=v$ ). After partial integration we writte the second term as

$$
\frac{1}{2 c_{w}}\left(H^{2}-v^{2}\right) Z_{\mu \nu} B^{\mu \nu}-\frac{1}{c_{w}}\left(H^{2}-v^{2}\right) Z_{\mu} \partial_{\nu} B^{\mu \nu} .
$$

In this last equation, the first term contains the vertex $h \gamma Z$ and it has to be subtracted using a combination of $\mathcal{O}_{B B}$ and $\mathcal{O}_{W W}$, see (4.19). In the second term in (4.29) we use the EoM (A.16). After some algebra one obtains

$$
\begin{aligned}
\mathcal{D}_{\kappa \gamma}= & i e R_{2} W_{\mu}^{+} W_{\nu}^{-}\left[A^{\mu \nu}-t_{w} Z^{\mu \nu}\right]+\left(\frac{h}{v} P_{1}\right)\left[\mathcal{W}_{\mu \nu}^{+} \mathcal{W}^{-\mu \nu}+\frac{c_{w}^{2}-s_{w}^{2}}{2 c_{w}^{2}} \mathcal{Z}_{\mu \nu} \mathcal{Z}^{\mu \nu}\right. \\
& \left.+t_{w}\left(\mathcal{A}_{\mu \nu} \mathcal{Z}^{\mu \nu}-A_{\mu \nu} Z^{\mu \nu}\right)+t_{w}^{2} Z_{\mu \nu} Z^{\mu \nu}\right]-\frac{g^{\prime 2}}{2 c_{w}^{2}}\left(h Q_{3}\right) v Z^{\mu} Z_{\mu} \\
& +2 \frac{g^{\prime} s_{w}}{c_{w}^{2}}\left(\frac{h}{v} P_{1}\right)\left(c_{w}^{2} J_{e m}^{\mu}-J_{Z}^{\mu}\right) Z_{\mu}
\end{aligned}
$$

where $P_{1}$ and $Q_{3}$ are given in (4.8) and (4.26), respectively, and

$$
R_{2}=1+\frac{2 h}{v}+\frac{h^{2}}{v^{2}}
$$

We have defined the direction (4.30) in a way that, when summed in (1.3), is to be multiplied by $\eta_{\kappa \gamma}=\delta \kappa_{\gamma}=-t_{w}^{-2} \delta \kappa_{Z}$.

We stress again that we choose the vertex $h Z_{\mu} Z^{\mu}$, appearing in the direction (4.30), as a custodial-breaking term. In addition, notice the appearance of terms $h Z_{\mu \nu} Z^{\mu \nu}$ and $h W_{\mu \nu} W^{\mu \nu}$, which contribute to $h \rightarrow V V^{(*)}$, also in a custodial-breaking combination. 
The $d=6$ operator in the coupling basis is

$$
\mathcal{O}_{\kappa \gamma}=\mathcal{O}_{H B}-\frac{1}{8}\left(\mathcal{O}_{W W}-\mathcal{O}_{B B}\right)
$$

It gives the desired direction $\mathcal{D}_{\kappa \gamma}$ provided that at the same time some redefinitions of SM parameters are made.

\subsection{3 $a=Z f f$}

Let us now turn our attention to operators with fermions. We start with the seven operators in (2.4), which can be related in a quite direct way to the seven couplings in (3.7) and (3.8).

The directions $a=Z f R, f=u, d, e$ in (3.7) are easily to obtain because $\mathcal{O}_{Z f R}=\mathcal{O}_{R}^{f}$, so that

$$
\begin{aligned}
& \mathcal{D}_{Z d R}=R_{2} Z_{\mu} \bar{d}_{R} \gamma^{\mu} d_{R}, \\
& \mathcal{D}_{Z u R}=R_{2} Z_{\mu} \bar{u}_{R} \gamma^{\mu} u_{R}, \\
& \mathcal{D}_{Z e R}=R_{2} Z_{\mu} \bar{e}_{R} \gamma^{\mu} e_{R} .
\end{aligned}
$$

Suitable combinations of $\mathcal{O}_{L}^{l}, \mathcal{O}_{L}^{(3) l}, \mathcal{O}_{L}^{q}, \mathcal{O}_{L}^{(3) q}$ give the $a=Z f L, f=u, d, \nu, e$, directions in (3.8), Indeed,

$$
\mathcal{O}_{Z u L}=\mathcal{O}_{L}^{(3) q}-\mathcal{O}_{L}^{q}, \quad \mathcal{O}_{Z d L}=\mathcal{O}_{L}^{(3) q}+\mathcal{O}_{L}^{q},
$$

and similarly for $e$ and $\nu$. We get

$$
\begin{aligned}
& \mathcal{D}_{Z u L}=R_{2}\left[Z_{\mu} \bar{u}_{L} \gamma^{\mu} u_{L}+\frac{c_{w}}{\sqrt{2}}\left(W_{\mu}^{+} \bar{u}_{L} \gamma^{\mu} d_{L}+\text { h.c. }\right)\right], \\
& \mathcal{D}_{Z d L}=R_{2}\left[Z_{\mu} \bar{d}_{L} \gamma^{\mu} d_{L}-\frac{c_{w}}{\sqrt{2}}\left(W_{\mu}^{+} \bar{u}_{L} \gamma^{\mu} d_{L}+\text { h.c. }\right)\right], \\
& \mathcal{D}_{Z e L}=R_{2}\left[Z_{\mu} \bar{e}_{L} \gamma^{\mu} e_{L}-\frac{c_{w}}{\sqrt{2}}\left(W_{\mu}^{+} \bar{\nu}_{L} \gamma^{\mu} e_{L}+\text { h.c. }\right)\right], \\
& \mathcal{D}_{Z \nu L}=R_{2}\left[Z_{\mu} \bar{\nu}_{L} \gamma^{\mu} \nu_{L}+\frac{c_{w}}{\sqrt{2}}\left(W_{\mu}^{+} \bar{\nu}_{L} \gamma^{\mu} e_{L}+\text { h.c. }\right)\right],
\end{aligned}
$$

where $R_{2}$ is given in (4.31).

\subsection{4 $a=D V f$ (dipoles)}

We now turn our attention to the dipole operators in (2.5) and the dipole couplings in (3.9). For the gluons, we simply have $\mathcal{O}_{D G q}=\mathcal{O}_{D G}^{q}$. Thus,

$$
\mathcal{D}_{D G q}=\left(\frac{P_{1}}{v}\right)\left[\bar{q}_{L} \sigma^{\mu \nu} T^{A} q_{R} \mathcal{G}_{\mu \nu}^{A}+\text { h.c. }\right]
$$

with $q=u, d$. Concerning the electroweak dipoles, we can reorganise the gauge invariant operators in (2.5) so that we obtain dipoles in the physical gauge-boson basis. We have, for the up-quark type

$$
\mathcal{O}_{D Z u}=\mathcal{O}_{D W}^{u}-\mathcal{O}_{D B}^{u}, \quad \mathcal{O}_{D A u}=\mathcal{O}_{D B}^{u}+t_{w}^{2} \mathcal{O}_{D W}^{u},
$$


and for down-type quark

$$
\mathcal{O}_{D Z d}=\mathcal{O}_{D W}^{d}+\mathcal{O}_{D B}^{d}, \quad \mathcal{O}_{D A d}=\mathcal{O}_{D B}^{d}-t_{w}^{2} \mathcal{O}_{D W}^{d},
$$

and similarly for the electron $e$. The corresponding directions $\mathcal{D}_{D V f}$ are

$$
\begin{aligned}
& \mathcal{D}_{D V u}=\left(\frac{P_{1}}{v}\right)\left[\bar{u}_{L} \sigma^{\mu \nu} u_{R} \mathcal{V}_{\mu \nu}+\sqrt{2} c_{V}\left(\bar{d}_{L} \sigma^{\mu \nu} u_{R} \mathcal{W}_{\mu \nu}^{-}+\text {h.c. }\right)\right], \\
& \mathcal{D}_{D V d}=\left(\frac{P_{1}}{v}\right)\left[\bar{d}_{L} \sigma^{\mu \nu} d_{R} \mathcal{V}_{\mu \nu}-\sqrt{2}\left(c_{V} \bar{d}_{L} \sigma^{\mu \nu} u_{R} \mathcal{W}_{\mu \nu}^{-}+\text {h.c. }\right)\right], \\
& \mathcal{D}_{D V e}=\left(\frac{P_{1}}{v}\right)\left[\bar{e}_{L} \sigma^{\mu \nu} e_{R} \mathcal{V}_{\mu \nu}-\sqrt{2}\left(c_{V} \bar{e}_{R} \sigma^{\mu \nu} \nu_{L} \mathcal{W}_{\mu \nu}^{-}+\text {h.c. }\right)\right] .
\end{aligned}
$$

Here $\mathcal{V}=\mathcal{Z}, \mathcal{A}$, as defined in (A.5), and $c_{Z}=c_{w}, c_{A}=s_{w}$. One may easily extend the results to three families.

\subsection{5 $a=u R d R$}

The operator in (2.6) leads to

$$
\mathcal{O}_{u R d R}=\mathcal{O}_{R}^{u d} \rightarrow H^{2} \bar{u}_{R} \gamma^{\mu} d_{R} W_{\mu}^{+}
$$

and to

$$
\mathcal{D}_{u R d R}=R_{2} \bar{u}_{R} \gamma^{\mu} d_{R} W_{\mu}^{+}+\text {h.c. }
$$

\subsection{TGC-only sector}

\subsection{1 $a=\lambda V$}

The operator (2.7) induces a TGC coupling accompanied by quartic interactions. The direction $\mathcal{O}_{\lambda V}=\mathcal{O}_{3 W}$ leads to

$$
\mathcal{D}_{\lambda V}=\frac{i}{m_{W}^{2}}\left(g c_{w} \mathcal{Z}^{\mu \nu}+e \mathcal{A}^{\mu \nu}\right) \mathcal{W}_{\nu}^{-\rho} \mathcal{W}_{\rho \mu}^{+} .
$$

The normalisation is $\eta_{\lambda V}=\lambda_{Z}=\lambda_{\gamma}$, in the parameterization in (3.5).

\section{The CP-odd directions}

The directions we associate to (2.9) are $a=h g \widetilde{g}, h \gamma \widetilde{\gamma}, h \gamma \widetilde{Z}$. To get them we use the decomposition (4.17). The terms with $v^{2}$ can be obviated, because they can be reabsorbed in the cases of $\mathcal{O}_{B \widetilde{B}}$ and $\mathcal{O}_{W \widetilde{W}}$, and in the case of $\mathcal{O}_{G \widetilde{G}}$ because it is a contribution to the $\theta$-QCD parameter, which we assume is rotated away with, for instance, a Peccei-Quinn mechanism. It follows that the operators (2.9) are of the "Higgs-only" CP-odd type.

The direction corresponding to the CP-odd coupling of Higgs to two gluons is simply given by $\mathcal{O}_{h g \widetilde{g}}=\mathcal{O}_{G \widetilde{G}}$, and from here we obtain

$$
\mathcal{D}_{h g \widetilde{g}}=\left(\frac{h}{v} P_{1}\right) \mathcal{G}_{\mu \nu}^{A} \widetilde{\mathcal{G}}^{A \mu \nu}
$$


The CP-odd coupling of Higgs to $\gamma \gamma, \gamma Z$ can be obtained with the simple linear combinations $\mathcal{O}_{h \gamma \widetilde{\gamma}}=\mathcal{O}_{B \widetilde{B}}+t_{w}^{2} \mathcal{O}_{W \widetilde{W}}$, and $\mathcal{O}_{h \gamma \widetilde{\gamma}}=\mathcal{O}_{h \gamma \widetilde{Z}}=\mathcal{O}_{B \widetilde{B}}-\mathcal{O}_{W \widetilde{W}}$. We get

$$
\begin{aligned}
& \mathcal{D}_{h \gamma \widetilde{\gamma}}=\left(\frac{h}{v} P_{1}\right)\left[\mathcal{A}_{\mu \nu} \widetilde{\mathcal{A}}^{\mu \nu}+\mathcal{Z}_{\mu \nu} \widetilde{\mathcal{Z}}^{\mu \nu}+2 \mathcal{W}_{\mu \nu}^{+} \widetilde{\mathcal{W}}^{-\mu \nu}\right] \\
& \mathcal{D}_{h \gamma \widetilde{Z}}=\left(\frac{h}{v} P_{1}\right)\left[\mathcal{A}_{\mu \nu} \widetilde{\mathcal{Z}}^{\mu \nu}+\frac{c_{w}^{2}-s_{w}^{2}}{2 c_{w} s_{w}} \mathcal{Z}_{\mu \nu} \widetilde{\mathcal{Z}}^{\mu \nu}+\frac{c_{w}}{s_{w}} \mathcal{W}_{\mu \nu}^{+} \widetilde{\mathcal{W}}^{-\mu \nu}\right] .
\end{aligned}
$$

The two operators in (2.10) contribute to the CP-odd TGC, see (3.12). Consider first $a=\widetilde{\kappa \gamma}$. When working out $\mathcal{O}_{H \widetilde{B}}$ in (2.10) one finds that the vertex corresponding to $h \gamma \widetilde{Z}$ has to be subtracted, like in the CP-even case in (4.29). However, a difference with the CP-even direction is that there are no more subtractions to be done because $\partial^{\nu} \widetilde{B}_{\mu \nu}=0$. As a result,

$$
\begin{aligned}
\mathcal{D}_{\widetilde{\kappa \gamma}}= & i e R_{2} W_{\mu}^{+} W_{\nu}^{-}\left[\widetilde{A}^{\mu \nu}-t_{w} \widetilde{Z}^{\mu \nu}\right]+\left(\frac{h}{v} P_{1}\right)\left[\mathcal{W}_{\mu \nu}^{+} \widetilde{\mathcal{W}}^{-\mu \nu}+\frac{c_{w}^{2}-s_{w}^{2}}{2 c_{w}^{2}} \mathcal{Z}_{\mu \nu} \widetilde{\mathcal{Z}}^{\mu \nu}\right. \\
& \left.+t_{w}\left(\mathcal{A}_{\mu \nu} \widetilde{\mathcal{Z}}^{\mu \nu}-A_{\mu \nu} \widetilde{Z}^{\mu \nu}\right)+t_{w}^{2} Z_{\mu \nu} \widetilde{Z}^{\mu \nu}\right]
\end{aligned}
$$

where the normalisation is such that $\eta_{\widetilde{\kappa} \gamma}=\delta \widetilde{\kappa}_{\gamma}$. The relation between bases in this case is $\mathcal{O}_{\widetilde{\kappa \gamma}}=\mathcal{O}_{H \widetilde{B}}-\left(\mathcal{O}_{W \widetilde{W}}-\mathcal{O}_{B \widetilde{B}}\right) / 8$

The contribution to $a=\widetilde{\lambda V}$ comes directly from $\mathcal{O}_{3 \widetilde{W}}$ in $(2.10)$, so that $\mathcal{O}_{\widetilde{\lambda V}}=\mathcal{O}_{3 \widetilde{W}}$ and

$$
\mathcal{D}_{\widetilde{\lambda V}}=\frac{i}{m_{W}^{2}}\left(g c_{w} \mathcal{Z}^{\mu \nu}+e \mathcal{A}^{\mu \nu}\right) \mathcal{W}_{\nu}^{-\rho} \widetilde{\mathcal{W}}_{\rho \mu}^{+}
$$

\section{The coupling basis}

In sections 4 and 5, for the chosen set of couplings $\{a\}$, we have determined the directions $\left\{\mathcal{D}_{a}\right\}$ and the linear combinations of the $\mathcal{O}_{i}$ 's we have to perform to get the coupling basis. Here we summarise all of them with a list of results in the form

$$
\mathcal{O}_{a}=\sum_{i} \kappa_{a}^{i} \mathcal{O}_{i} \triangleright \kappa_{a} \mathcal{D}_{a}
$$

where $\kappa_{a}^{i}$ shows the required particular combination for each $a$, and $\kappa_{a}$ gives the normalisation. The symbol $\triangleright$ means that we take the expressions for $\mathcal{O}$ in the unitary gauge and that, when needed, we redefine some SM bare parameters.

We first list the operators that do no involve fermions (even if fermions are present in some directions),

$$
\begin{aligned}
\mathcal{O}_{h 3} & =\mathcal{O}_{6} & & \triangleright \lambda v^{2} \mathcal{D}_{h 3}, \\
\mathcal{O}_{h(V V)_{c}} & =\mathcal{O}_{r}-\frac{1}{2} \mathcal{O}_{6} & & \triangleright \frac{g^{2}}{4} v^{2} \mathcal{D}_{h(V V)_{c}}, \\
\mathcal{O}_{h g g} & =\mathcal{O}_{G G} & & \triangleright g_{s}^{2} v^{2} \mathcal{D}_{h g g}, \\
\mathcal{O}_{h \gamma \gamma} & =\mathcal{O}_{B B}+t_{w}^{2} \mathcal{O}_{W W} & & \triangleright g^{\prime 2} v^{2} \mathcal{D}_{h \gamma \gamma},
\end{aligned}
$$




$$
\begin{array}{rlrl}
\mathcal{O}_{h \gamma Z} & =\mathcal{O}_{W W}-\mathcal{O}_{B B} & \triangleright 2 g g^{\prime} v^{2} \mathcal{D}_{h \gamma Z}, \\
\mathcal{O}_{g 1 Z}=\mathcal{O}_{B}-\mathcal{O}_{W}-g^{2}\left(\mathcal{O}_{r}-\frac{1}{2} \mathcal{O}_{6}\right) & \triangleright \frac{g^{2}}{4 c_{w}^{2}} v^{2} \mathcal{D}_{g 1 Z}, \\
\mathcal{O}_{\kappa \gamma}=\mathcal{O}_{H B}-\frac{1}{8}\left(\mathcal{O}_{W W}-\mathcal{O}_{B B}\right) & \triangleright-\frac{g^{2}}{4} v^{2} \mathcal{D}_{\kappa \gamma}, \\
\mathcal{O}_{\lambda V}=\mathcal{O}_{3 W} & \triangleright \frac{g^{2}}{4} v^{2} \mathcal{D}_{\lambda V},
\end{array}
$$

and operators with fermions

$$
\begin{array}{rlrl}
\mathcal{O}_{h f f} & =\mathcal{O}_{y_{f}} & & \frac{y_{f}}{\sqrt{2}} v^{2} \mathcal{D}_{h f f}, \\
\mathcal{O}_{Z f R} & =\mathcal{O}_{R}^{f} & & -\frac{g}{2 c_{w}} v^{2} \mathcal{D}_{Z f R}, \\
\mathcal{O}_{Z f L} & =\mathcal{O}_{L}^{(3) F}-\left(2 T_{3}^{f}\right) \mathcal{O}_{L}^{F} & & \left(2 T_{3}^{f}\right) \frac{g}{c_{w}} v^{2} \mathcal{D}_{Z f L}, \\
\mathcal{O}_{D G q} & =\mathcal{O}_{D G}^{q} & & y_{q} g_{s} v^{2} \mathcal{D}_{D G q}, \\
\mathcal{O}_{D Z f} & =\mathcal{O}_{D W}^{f}-\left(2 T_{3}^{f}\right) \mathcal{O}_{D B}^{f} & \triangleright & \left(2 T_{3}^{f}\right) y_{f} \frac{g}{c_{w}} v^{2} \mathcal{D}_{D Z f}, \\
\mathcal{O}_{D A f} & =\mathcal{O}_{D B}^{f}+\left(2 T_{3}^{f}\right) t_{w}^{2} \mathcal{O}_{D W}^{f} & \triangleright y_{f} \frac{g^{\prime}}{c_{w}} v^{2} \mathcal{D}_{D A f}, \\
\mathcal{O}_{u R d R}=\mathcal{O}_{R}^{u d} & & \frac{y_{u} y_{d}}{\sqrt{2}} g^{2} v^{2} \mathcal{D}_{u R d R} .
\end{array}
$$

Here, $f=u, d, e$ (and also $\nu$ in $Z f L$ ), $F=q, l$, and $q=u, d$. The weak isospin of $f$ is denoted by $T_{3}^{f}$. In the dipole operators, the operators $\mathcal{O}_{i}$ are understood to be summed with its hermitian conjugate.

The CP-odd list is

$$
\begin{array}{rlrl}
\mathcal{O}_{h g \widetilde{g}} & =\mathcal{O}_{G \widetilde{G}} & & g_{s}^{2} v^{2} \mathcal{D}_{h g \widetilde{g}}, \\
\mathcal{O}_{h \gamma \widetilde{\gamma}} & =\mathcal{O}_{B \widetilde{B}}+t_{w}^{2} \mathcal{O}_{W \widetilde{W}} & & g^{2} v^{2} \mathcal{D}_{h \gamma \widetilde{\gamma}}, \\
\mathcal{O}_{h \gamma \widetilde{Z}} & =\mathcal{O}_{W \widetilde{W}}-\mathcal{O}_{B \widetilde{B}} & \triangleright & 2 g g^{\prime} v^{2} \mathcal{D}_{h \widetilde{\gamma} Z}, \\
\mathcal{O}_{\widetilde{\kappa \gamma}}=\mathcal{O}_{H \widetilde{B}}-\frac{1}{8}\left(\mathcal{O}_{W \widetilde{W}}-\mathcal{O}_{B \widetilde{B}}\right) & \triangleright-\frac{g^{2}}{4} v^{2} \mathcal{D}_{\widetilde{\kappa \gamma}}, \\
\mathcal{O}_{\widetilde{\lambda V}}=\mathcal{O}_{3 \widetilde{W}} & \triangleright \frac{g^{2}}{4} v^{2} \mathcal{D}_{\widetilde{\lambda V}} .
\end{array}
$$

We stress that the expressions for the $\mathcal{D}_{a}$ are unique, up to partial integrations and redefinitions. The particular linear combinations that lead from $\left\{\mathcal{O}_{i}\right\}$ to $\left\{\mathcal{O}_{a}\right\}$ depend of course on the particular starting basis we have chosen. Just as an example, if in our starting basis we had the operator $\mathcal{O}_{H}=\frac{1}{2}\left(\partial^{\mu}|\Phi|^{2}\right)^{2}$ instead of $\mathcal{O}_{r}$, we could also get the $h(V V)_{c}$ direction in 4.1.3, but using the combination

$$
\mathcal{O}_{h(V V)_{c}}=\mathcal{O}_{H}-\sum_{f} \frac{1}{2} \mathcal{O}_{y_{f}}-\frac{3}{2} \mathcal{O}_{6} \triangleright-\frac{g^{2}}{4} v^{2} \mathcal{D}_{h(V V)_{c}}
$$

We see that the combinations required to get $a=h(V V)_{c}$ in the case of $\mathcal{O}_{r}$ are simpler than in the case of $\mathcal{O}_{H}$, and this is why we opted for the first. This is an example of choosing the starting basis as "close" as possible to $\left\{\mathcal{O}_{a}\right\}$. 
A final comment is that we have systematically eliminated the presence of terms with derivatives acting on the Higgs field. In our case, this can be done using partial integration and applying EoMs.

\section{Discussion and conclusions}

The correlations induced by gauge-invariant operators in effective Lagrangians have interesting phenomenological consequences. In the case of observing a deviation from a SM prediction -sign of BSM-, such correlations imply that deviations on some other couplings should be found. By the same token, constraints on deviations from the SM in some couplings imply constraints on other couplings. The correlations might be a very useful guide to BSM physics. However, the analysis is complicated by the fact that, in general, each operator contributes to different couplings and, in turn, each coupling receives constraints from several operators.

In this paper we have shown how to write the effective Lagrangian in a basis where each independent gauge-invariant operator $\mathcal{O}_{a}$ is linked to a particular coupling $a$, described by a term $\widehat{\mathcal{D}}_{a}$ multiplied by $\eta_{a}$, the potential deviation from the SM prediction. In a sense, our work has been to "complete" $\widehat{\mathcal{D}}_{a}$ with other structures to have finally a gauge-invariant operator $\mathcal{O}_{a}$. Each term $\widehat{\mathcal{D}}_{a}$ appears once, and only once, in the effective Lagrangian. With this requirements, the expressions for $\mathcal{O}_{a}$ and $\mathcal{D}_{a}$ are unique, up to partial integrations, redefinitions etc.

The number of couplings $\{a\}$ in the sum (1.3) equals the number of operators of a basis in operator space. We restrict our study to physics relevant for EWPT, TGC and Higgs physics. Then this number is equal to 27 (in the CP-even sector, for one family) and 5 (in the CP-odd sector). This makes a total of 32 . We have chosen the couplings that are better measured or eventually will be. In the case that in the future the experimental situation changes, we could easily choose another set of couplings and work out a form of the effective Lagrangian suited to this novel group.

In tables 1,2 and 3 we list all the couplings in the set $\{a\}$, together with the defining operator $\widehat{\mathcal{D}}_{a}$. The complete direction is $\mathcal{D}_{a}=\widehat{\mathcal{D}}_{a}+\delta \mathcal{D}_{a}$. In the tables we show schematically which couplings one has for each $a$, up to four-particle vertices. Given two different couplings $a$ and $b$ we may ask whether there are some common terms in $\delta \mathcal{D}_{a}$ and $\delta \mathcal{D}_{b}$. This has physical interest, because a common term in two directions means the existence of a correlation. More generally, we may separate our set of couplings $\{a\}$ in groups or classes, such that inside a group each direction has at least a common term with at least another direction of the group. In other words, a direction in a group has no common term with any other direction of the other groups. This splitting leads to a group of 16 (CP-even) directions shown in table 1 . The 11 remaining $\mathrm{CP}$-even directions are split in 7 classes, indicated in table 2. Finally, in table 3 we show the 5 CP-odd directions, separated in 4 classes.

A first good property of our approach has to do with what we have just discussed: in the search for deviations from the SM predictions, our effective Lagrangian (1.3) may 


\begin{tabular}{|c|c|c|}
\hline$a$ & $\widehat{\mathcal{D}}_{a}$ & $\delta \mathcal{D}_{a}$ \\
\hline$h f f(3)$ & $h \bar{f} f$ & $h^{2} \bar{f} f$ \\
\hline$h 3$ & $h^{3}$ & $h^{4}$ \\
\hline$h(V V)_{c}$ & $v h\left(W^{+\mu} W_{\mu}^{-}+\frac{1}{2 c_{w}^{2}} Z^{\mu} Z_{\mu}\right)$ & $h \widehat{\mathcal{D}}_{h(V V)_{c}}, h^{2} \bar{f} f, h^{4}$. \\
\hline$h \gamma \gamma$ & $\frac{1}{v} h A^{\mu \nu} A_{\mu \nu}$ & $\begin{array}{c}h \widehat{\mathcal{D}}_{h \gamma \gamma}, h W^{2} \partial V_{\gamma, Z} \\
h W V_{\gamma, Z} \partial W, h^{1,2}\left(\partial V_{Z, W}\right)^{2}\end{array}$ \\
\hline$h \gamma Z$ & $\frac{1}{v} h A^{\mu \nu} Z_{\mu \nu}$ & $\begin{array}{c}h \widehat{\mathcal{D}}_{h \gamma Z}, h W^{2} \partial V_{\gamma, Z} \\
h W V_{\gamma, Z} \partial W, h^{1,2}\left(\partial V_{Z, W}\right)^{2}\end{array}$ \\
\hline$g 1 Z$ & $\begin{aligned} i g c_{w} & {\left[Z^{\mu}\left(W^{+\nu} W_{\mu \nu}^{-}-\text {h.c }\right)\right.} \\
+ & \left.Z^{\mu \nu} W_{\mu}^{+} W_{\nu}^{-}\right]\end{aligned}$ & $\begin{array}{c}Z W^{2} V_{\gamma, Z}, W^{4}, h^{2}(V V)_{c}, h^{1,2}(V V)_{n c} \\
h^{2} \bar{f} f, h^{4}, h Z J_{R, L}, h W J_{L}\end{array}$ \\
\hline$\kappa \gamma$ & $i e W_{\mu}^{+} W_{\nu}^{-}\left(A^{\mu \nu}-t_{w} Z^{\mu \nu}\right)$ & $\begin{array}{c}h \widehat{\mathcal{D}}_{\kappa \gamma}, h W^{2} \partial V_{\gamma, Z}, h V_{\gamma, Z} W \partial W \\
h^{1,2}\left(\partial V_{Z, W}\right)^{2}, h^{1,2}(V V)_{n c}, h Z J_{R, L}\end{array}$ \\
\hline$Z f R$ & $Z_{\mu} \bar{f}_{R} \gamma^{\mu} f_{R}$ & $h \widehat{\mathcal{D}}_{Z f R}$ \\
\hline$Z f L$ & $Z_{\mu} \bar{f}_{L} \gamma^{\mu} f_{L}$ & $h \widehat{\mathcal{D}}_{Z f L} \quad, \quad h W J_{L}$ \\
\hline
\end{tabular}

Table 1. For each direction $a$ and $\widehat{\mathcal{D}}_{a}$ we show the couplings in $\delta \mathcal{D}_{a}$ involving three or four particles. We display them schematically, for example $\partial V$ stands for the $V_{\mu \nu}$ field strength, $V_{\gamma, Z}$ for $A_{\mu}$ or $Z_{\mu}, Z J_{R}$ for $Z_{\mu} \bar{f}_{R} \gamma^{\mu} f_{R}, h^{1,2}$ for $h$ and $h^{2}$, etc. We write the non-custodial coupling $h Z^{\mu} Z_{\mu}$ as $h(V V)_{n c}$. The exact form of the couplings as well as the factor in front are given in the formulas in the text. The 16 directions in the table form a group with the following property. Any of them has at least one common term with at least another of direction in the group. In the first column, the number is parenthesis corresponds to the number of directions, when greater than one (for one fermion family). In $a=h f f, Z f R, f=u, d, e$; in $a=Z f L, f=u, d, e, \nu$ The dashed lines separate directions measured in $h$-physics, TGC and $Z$-physics.

facilitate the analyses looking for BSM signals. There are more advantages of our approach that we now comment.

In general, effective Lagrangians induce corrections to the fine structure constant $\alpha$, and to masses of particles in the SM. When we use, for example $\alpha$ and $m_{Z}$ in the set of input parameters, those corrections propagate to the predictions of the effective Lagrangian. (These effects were called indirect corrections in [32].) They appear because the predictions of the SM have to be expressed as functions of $\alpha, m_{Z}$, etc. In this regard, a positive aspect of our approach is that all the terms in our $\mathcal{L}_{6}$ are vertices containing three or more particles. This means that there are no corrections to tree-level masses. Also, there is no correction to $\alpha$. The form of our effective Lagrangian makes the use of $\alpha, m_{Z}, m_{W}, m_{h}$ and $m_{f}$ as input parameters very convenient. To use $m_{W}$ instead of $G_{F}$ is simpler, because the use of the latter involves a four-fermion operator. Besides, the $m_{W}$ precision has reached an accuracy that allows to use its measurement as input. When we use $\alpha$ and particles masses 


\begin{tabular}{|c|c|c|}
\hline$a$ & $\widehat{\mathcal{D}}_{a}$ & $\delta \mathcal{D}_{a}$ \\
\hline$h g g$ & $\frac{1}{v} h G^{A \mu \nu} G_{\mu \nu}^{A}$ & $h \widehat{\mathcal{D}}_{g g}, h G^{2} \partial G$. \\
\hline$\lambda V$ & $\frac{i}{m_{W}^{2}}\left(g c_{w} Z^{\mu \nu}+e A^{\mu \nu}\right) W_{\nu}^{-\rho} W_{\rho \mu}^{+}$ & $W^{2}(\partial W)^{2}, V_{\gamma, Z} W \partial V_{\gamma, Z} \partial W$. \\
\hline$D G u$ & $\frac{1}{v} \bar{u} \sigma^{\mu \nu} T^{A} u G_{\mu \nu}^{A}$ & $h \widehat{\mathcal{D}}_{D G u} \cdot$ \\
\hline$D G d$ & $\frac{1}{v} \bar{d} \sigma^{\mu \nu} T^{A} d G_{\mu \nu}^{A}$ & $h \widehat{\mathcal{D}}_{D G d} \cdot$ \\
\hline$D V q(4)$ & $\frac{1}{v} \bar{q} \sigma^{\mu \nu} q V_{\mu \nu}$ & $h \widehat{\mathcal{D}}_{D V q},\left(\bar{q} \sigma^{\mu \nu} q\right) W_{\mu}^{+} W_{\nu}^{-}$, \\
& $\frac{1}{v}\left(\bar{e} \sigma^{\mu \nu} e\right) V_{\mu \nu}\left(\bar{u} \sigma^{\mu \nu} d\right) W_{\mu \nu},\left(\bar{u} \sigma_{\mu \nu} d\right) W^{\mu} V_{\gamma, Z}^{\nu} \cdot$ \\
\hline$D V e(2)$ & $h \widehat{\mathcal{D}}_{D V e},\left(\bar{e} \sigma^{\mu \nu} e\right) W_{\mu}^{+} W_{\nu}^{-}$, \\
& $h^{0,1}\left(\bar{e} \sigma^{\mu \nu} \nu\right) W_{\mu \nu},\left(\bar{e} \sigma_{\mu \nu} \nu\right) W^{\mu} V_{\gamma, Z}^{\nu} \cdot$ \\
\hline$u R d R$ & $\bar{u}_{R} \gamma^{\mu} d_{R} W_{\mu}^{+}$ & $h \widehat{\mathcal{D}}_{u R d R} \cdot$ \\
\hline
\end{tabular}

Table 2. Same than table 1 , for the rest of CP-even directions. Here the split is in 7 groups. In $a=D V q, D V e$ we have $q=u, d, V=A, Z$.

\begin{tabular}{|c|c|c|}
\hline$a$ & $\widehat{\mathcal{D}}_{a}$ & $\delta \mathcal{D}_{a}$ \\
\hline$h g \widetilde{g}$ & $\frac{1}{v} h G^{A \mu \nu} \widetilde{G}_{\mu \nu}^{A}$ & $h \widehat{\mathcal{D}}_{g \widetilde{g}}, h G^{2} \widetilde{\partial G}$. \\
\hline$h \gamma \widetilde{\gamma}$ & $\frac{1}{v} h A^{\mu \nu} \widetilde{A}_{\mu \nu} \widetilde{\mathcal{D}}_{h \gamma \widetilde{\gamma}}, h W^{2} \widetilde{\partial V}_{\gamma, Z}$, \\
$h \gamma \widetilde{Z}$ & $h W V_{\gamma, Z} \widetilde{\partial W}, h^{1,2} \partial V_{Z, W} \widetilde{\partial V} \widetilde{Z}_{Z, W} \cdot$ \\
& $h \widehat{\mathcal{D}}_{h \gamma \widetilde{Z}}, h W^{2} \widetilde{\partial V} \widetilde{\gamma}_{\gamma, Z}$, \\
$\widetilde{\kappa \gamma}$ & $i e W_{\mu}^{+} W_{\nu}^{-}\left(\widetilde{A}^{\mu \nu}-t_{w} \widetilde{Z}^{\mu \nu}\right)$ & $h \widetilde{Z}_{\gamma, Z} \widetilde{\partial W}, h^{1,2} \partial V_{Z, W} \widetilde{\partial V} \widetilde{Z}_{Z, W} \cdot$ \\
\hline$\widetilde{\lambda V}$ & $\frac{i}{m_{W}^{2}}\left(g c_{w} Z^{\mu \nu}+e A^{\mu \nu}\right) W_{\nu}^{-\rho} \widetilde{W}_{\rho \mu}^{+}$ & $W^{2} \partial W \widetilde{\partial W}, h W^{2} \partial \widetilde{V}_{\gamma, Z}$, \\
\hline
\end{tabular}

Table 3. Same than table 1, for the CP-odd directions.

as input, we do not have indirect corrections, and thus calculations using the form of our effective Lagrangian are simplified.

We should also mention the problem of blind directions [32]. It may happen that in a certain basis there is a linear combination of operators that experiments cannot bound, i.e., a blind direction. By definition, in our approach there are no blind directions.

Even if is not the purpose of this paper to look for concrete applications of our approach, it may be useful to sketch ideas about some possible applications. As we said, in the case of measuring a deviation from the SM prediction in a certain coupling $a$, one can immediately see in $\delta \mathcal{D}_{a}$ which vertices should show a positive signal, and with what strength. Of course we knew there are correlations, but the expressions we get in our framework for the directions show them in a transparent way. 
Another aspect, complementary of what we have just explained, is that our results show when there is no correlation. Let us explain it with an example. The LHC is expected to improve the LEP-2 measurements on TGC. Suppose that there is no sign of new physics, and thus LHC improves the constraints on TGC. We may ask the question: is this going to tell us something about $h \rightarrow \gamma \gamma$ or $h \rightarrow \gamma Z$ decays? We cite this example because in a general basis, for example the one in [27], one has operators contributing both to TGC and these Higgs decays, so that it is difficult to see whether there are correlations or not. Thanks to our formalism, we can easily see that the TGC directions are completely independent of the $h \gamma \gamma$ and the $h \gamma Z$ directions, so that the answer to the preceding question is a clear no.

Let us briefly mention other possible applications. One of the uses of effective Lagrangians is the power to anticipate a constraint on a process using current limits on couplings. Which are the relevant couplings and which one (or ones) is the dominant one can be readily seen in the expressions for our directions. A simple example will help to clarify this point. In the effective Lagrangian there is a contact term $h V_{\mu} \bar{f} \gamma^{\mu} f$, with $V=Z, W$ which contributes to the decays $h \rightarrow V f f$ measured at LHC. Our results show that such a term appears in the directions $a=Z f R, Z f L, g 1 Z, \kappa \gamma$. Then the anticipated constraint on $h V f f$ comes from these terms. In addition, since the limits on $\eta_{g 1 Z}, \eta_{\kappa \gamma}$ are order percent while on $Z f R, Z f L$ are order permille, it is the former that sets the magnitude of the constraint to be expected for $h V f f$. This was already pointed out in [24]. Another example is the custodial breaking terms $h Z^{\mu} Z_{\mu}$ appearing only in the directions $g 1 Z, \kappa \gamma$. The limits on this two TGC directions put a limit to the expected breaking of custodial symmetry in the $h V^{\mu} V_{\mu}, V=Z, W$, amplitudes. There are also custodial breaking effects in the couplings $h V^{\mu \nu} V_{\mu \nu}$, which appear in the directions $a=h \gamma Z, \kappa \gamma$. An interesting final example refers to the quartic gauge couplings, which are starting being measured at LHC. In our work, we show precisely how the modifications to the quartic gauge couplings are linked to the TGC parameters $g_{1}^{Z}, \kappa_{\gamma}$ and $\lambda_{Z}=\lambda_{\gamma}$.

Very recently, the Lagrangian (1.6) was deduced in [25]. The authors build it in a bottom-up approach: they make use of symmetries and analyze field structures to find all the independent terms in the effective Lagrangian, and generate as we have done all directions corresponding to couplings. In [25], emphasis is done on the fact that (1.3) helps in the search of BSM physics, as we do in our paper. There, the directions are called BSM Primary Effects. The final results in [25] and the ones found here agree, as expected. The two methods are very different since we use a top-down approach. Indeed, we start with the full relevant set of $d=6$ operators and manipulate them to get the directions. In a sense, our method is more systematic. As we said, in the future we could need to change the group of well-measured couplings. In this eventuality, we think our top-down approach is more suitable for such a change.

We end with a couple of remarks. First, our analytical results are not meant to be a substitute of a dedicated numerical investigation, necessary to be able to state limits with a certain CL, etc. The Lagrangian we have reached helps us in the understanding of some general properties, but of course can be perfectly used for numerical analyses. Second, our findings assume the framework of a linear effective Lagrangian with $d=6$ operators. 
In the case that the predicted correlations are not observed, this would mean that such a framework is not valid, which would also be precious information on BSM.

\section{A SM Lagrangian notation}

Here we write the EW SM Lagrangian, in the unitary gauge, in order to fix some notation. We use the following conventions. For the covariant derivative

$$
D_{\mu}=\partial_{\mu}-i g T^{a} W_{\mu}^{a}-i g^{\prime} Y B_{\mu}
$$

where $T^{a}=\sigma^{a} / 2$ for $\mathrm{SU}(2)$ doublets. The Higgs field $\left(Y_{\Phi}=1 / 2\right)$, in the unitary gauge, reads

$$
\Phi=\frac{1}{\sqrt{2}}\left(\begin{array}{c}
0 \\
H
\end{array}\right)=\frac{1}{\sqrt{2}}\left(\begin{array}{c}
0 \\
v+h
\end{array}\right) .
$$

Here $H / \sqrt{2}$ is the neutral part of the Higgs doublet, $h$ is the physical Higgs field.

We write the Lagrangian in the unitary gauge as

$$
\mathcal{L}_{S M}=\mathcal{L}_{G}+\mathcal{L}_{f}+\mathcal{L}_{G f}+\mathcal{L}_{H}
$$

The pure gauge and gauge-Higgs part is

$$
\begin{aligned}
\mathcal{L}_{G}= & -\frac{1}{4} \mathcal{A}^{\mu \nu} \mathcal{A}_{\mu \nu}-\frac{1}{4} \mathcal{Z}^{\mu \nu} \mathcal{Z}_{\mu \nu}-\frac{1}{2} \mathcal{W}^{+\mu \nu} \mathcal{W}_{\mu \nu}^{-} \\
& +\frac{1}{4} g^{2} H^{2} W^{+\mu} W_{\mu}^{-}+\frac{1}{8} \frac{g^{2}}{c_{w}^{2}} H^{2} Z^{\mu} Z_{\mu} .
\end{aligned}
$$

We have defined

$$
\begin{aligned}
\mathcal{A}_{\mu \nu} & =A_{\mu \nu}+i g s_{w}\left(W_{\mu}^{-} W_{\nu}^{+}-W_{\mu}^{+} W_{\nu}^{-}\right), \\
\mathcal{Z}_{\mu \nu} & =Z_{\mu \nu}+i g c_{w}\left(W_{\mu}^{-} W_{\nu}^{+}-W_{\mu}^{+} W_{\nu}^{-}\right), \\
\mathcal{W}_{\mu \nu}^{ \pm} & =W_{\mu \nu}^{ \pm} \pm i g\left(W_{\mu}^{ \pm} W_{\nu}^{3}-W_{\mu}^{3} W_{\nu}^{ \pm}\right),
\end{aligned}
$$

with $V_{\mu \nu}=\partial_{\mu} V_{\nu}-\partial_{\nu} V_{\mu}$ (what we denote by $A_{\mu \nu}$ is usually denoted by $F_{\mu \nu}$ ). We write the Lagrangian in terms of the weak bosons $W^{ \pm}, Z$ and the photon $A$, and for that purpose we introduce

$$
s_{w}=\sin \theta_{w}=\frac{g^{\prime}}{\sqrt{g^{2}+g^{\prime 2}}}, \quad c_{w}=\cos \theta_{w}=\frac{g}{\sqrt{g^{2}+g^{\prime 2}}}, \quad t_{w}=\tan \theta_{w} .
$$

For example, in the definition of $\mathcal{W}$ in (A.5), we have to substitute $W_{\mu}^{3}=c_{w} Z_{\mu}+s_{w} A_{\mu}$.

The fermion and Higgs-fermion terms in (A.3) are given by

$$
\mathcal{L}_{f}=i \bar{f} \not \partial f-\frac{y_{f}}{\sqrt{2}} H \bar{f} f
$$

where $y_{f}$ are the Yukawa couplings and where we understand a sum over fermions. In (A.3) we also have the gauge boson-fermion interactions

$$
\mathcal{L}_{G f}=\frac{g}{\sqrt{2}}\left(J_{W}^{\mu} W_{\mu}^{+}+\text {h.c. }\right)+\frac{g}{c_{w}} J_{Z}^{\mu} Z_{\mu}+e J_{e m}^{\mu} A_{\mu} .
$$


We define the currents

$$
J^{a \mu}=\bar{F}_{L} \frac{\sigma^{a}}{2} \gamma^{\mu} F_{L} \quad, \quad J_{Y}^{\mu}=\bar{f} Y_{f} \gamma^{\mu} f,
$$

where $F_{L}$ are the fermion doublets, and again a sum over fermions is understood. The currents appearing in (A.8) are

$$
\begin{aligned}
J_{W}^{\mu} & =J^{1 \mu}+i J^{2 \mu}, \\
J_{Z}^{\mu} & =c_{w}^{2} J^{3 \mu}-s_{w}^{2} J_{Y}^{\mu}, \\
J_{e m}^{\mu} & =J^{3 \mu}+J_{Y}^{\mu}=\bar{f} Q_{f} \gamma^{\mu} f
\end{aligned}
$$

Finally, the pure $H$-part is

$$
\mathcal{L}_{H}=\frac{1}{2}\left(\partial^{\mu} H\right)\left(\partial_{\mu} H\right)-V(H)
$$

where the potential

$$
V(H)=\frac{\lambda}{4}\left(H^{2}-v^{2}\right)^{2}=-\frac{\mu^{2}}{2} H^{2}+\frac{\lambda}{4} H^{4}+\text { constant }
$$

We take as input parameters $\alpha$ and the masses $m_{Z}, m_{W}, m_{h}$, and $m_{f}$. Parameters appearing in the Lagrangian are functions of these input parameters, for example

$$
c_{w}=\frac{m_{W}}{m_{Z}} \simeq 0.88, \quad v^{2}=\frac{m_{W}^{2}}{\pi \alpha}\left(1-\frac{m_{W}^{2}}{m_{Z}^{2}}\right) \simeq(243 \mathrm{GeV})^{2},
$$

where we have used $\alpha^{-1}\left(m_{Z}^{2}\right) \simeq 129$ [33]. We also have

$$
g=\frac{e}{s_{w}}, \quad y_{f}=\frac{\sqrt{2} m_{f}}{v}, \lambda=\frac{m_{h}^{2}}{2 v^{2}}, \quad \mu^{2}=\frac{m_{h}^{2}}{2} .
$$

In our calculations, we need the EoM for the Higgs field,

$$
\begin{aligned}
\partial^{\mu} \partial_{\mu} H= & \frac{g^{2}}{2} H W^{+\mu} W_{\mu}^{-}+\frac{g^{2}}{4 c_{w}^{2}} H Z^{\mu} Z_{\mu} \\
& -\frac{y_{f}}{\sqrt{2}} \bar{f} f-\lambda\left(H^{2}-v^{2}\right) H,
\end{aligned}
$$

and the EoMs for the gauge bosons in the $W_{\mu}^{a}, B_{\mu}$ basis,

$$
\begin{aligned}
D^{\nu} \mathcal{W}_{\mu \nu}^{a} & =\partial^{\nu} \mathcal{W}_{\mu \nu}^{a}+g \epsilon^{a b c} W^{b \nu} \mathcal{W}_{\mu \nu}^{c} \\
& =\frac{g}{4}\left(g W_{\mu}^{a}-g^{\prime} \delta^{a 3} B_{\mu}\right) H^{2}+g J_{\mu}^{a}, \\
\partial^{\nu} B_{\mu \nu} & =-\frac{g^{\prime}}{4}\left(g W_{\mu}^{3}-g^{\prime} B_{\mu}\right) H^{2}+g^{\prime} J_{\mu}^{Y},
\end{aligned}
$$

where $\mathcal{W}_{\mu \nu}^{a}$ and $B_{\mu \nu}$ are the $\mathrm{SU}(2)_{L}$ and $\mathrm{U}(1)_{Y}$ field strengths. 


\section{B Our starting basis and the basis of Grzadkowski et al.}

The first time a complete basis of $d=6$ operators was presented in the literature was in [27]. The basis consists of 59 operators (for one family), with 53 CP-even and 6 CP-odd. We now show that our counting and our basis are consistent with the basis in [27]. We start with the CP-even operators.

Of the 53 CP-even operators in the basis in [27], we do not consider the operator $\mathcal{G}^{3}$ -the analogous of (2.7) with the $\mathrm{SU}(3)$ field strength- because it contains exclusively gluon fields. Also, we do not consider the 25 four-fermion operators, because they do not play a role in our analysis. This leaves us with $53-1-25=27$ operators, which is the same number that we have, see (2.8).

We demonstrate now that the two basis of 27 operators are equivalent. To do it, we follow the classification nomenclature of [27], see their table 2, and compare with our set of operators. We identify operators that differ only in numerical factors, couplings and signs.

In the group $X^{3}$, only $\mathcal{O}_{3 W}$ matters. Other operators in this group are CP-odd and/or contain only gluons. The operator $\mathcal{O}_{3 W}$ is in our basis, see (2.7). There are three operators in the group $\phi^{6}$ and $\phi^{4} D^{2}: \mathcal{O}_{6}$, which in our list is $(2.1)$, and two which are not in our basis,

$$
\mathcal{O}_{T}=\frac{1}{2}\left(\Phi^{\dagger} \stackrel{\leftrightarrow}{D}_{\mu} \Phi\right)^{2} \quad, \quad \mathcal{O}_{H}=\frac{1}{2}\left(\partial^{\mu}|\Phi|^{2}\right)^{2}
$$

Next group in table 2 is $\psi^{2} \phi^{3}$. All three operators in this group are in our basis, they are $\mathcal{O}_{y_{f}}$ in (2.2). In the group $X^{2} \phi^{2}$ there are four CP-even operators. Three are in our list: $\mathcal{O}_{G G}, \mathcal{O}_{B B}, \mathcal{O}_{W W}$, see $(2.1)$, but not the fourth one:

$$
\mathcal{O}_{W B}=g^{\prime} g\left(\Phi^{\dagger} \sigma^{a} \Phi\right) \mathcal{W}_{\mu \nu}^{a} B^{\mu \nu} .
$$

The dipole group in [27], denoted by $\psi^{2} X \phi$, contains eight operators. We have these eight dipole operators, $\mathcal{O}_{D V}^{f}$ in (2.5). Finally in the group $\psi^{2} \phi^{2} D$ in table 2 of [27] there are eight operators. Seven of them correspond to our seven operators in (2.4) and the eighth is our (2.6).

As we said, the total number of $C P$-even operators extracted from [27] which are relevant for our study is 27 , which is precisely the number we have. We have seen that we have all of them except the three operators in (B.1) and (B.2). Instead of these three we have

$$
\mathcal{O}_{r}, \quad \mathcal{O}_{B}-\mathcal{O}_{W}, \quad \mathcal{O}_{H B}
$$

The way to relate our three operators in (B.3) to the three operators in (B.1) and (B.2) is using field redefinitions, as explained for example in [23]. We can use the relations

$$
\begin{aligned}
\mathcal{O}_{H} & \leftrightarrow-\mathcal{O}_{r}+\Lambda_{1}\left(\mathcal{O}_{y_{f}}, \mathcal{O}_{6}\right) \\
g^{\prime 2} \mathcal{O}_{T} & \leftrightarrow-2\left(\mathcal{O}_{B}-\mathcal{O}_{W}\right)+\Lambda_{2}\left(\mathcal{O}_{H}, \mathcal{O}_{6}, \mathcal{O}_{y_{f}}, \mathcal{O}_{L}^{(3) f}, \mathcal{O}_{L}^{f}, \mathcal{O}_{R}^{f}\right) \\
\frac{1}{4} \mathcal{O}_{W B} & \leftrightarrow-\mathcal{O}_{H B}+\left(\mathcal{O}_{B}-\mathcal{O}_{W}\right)+\Lambda_{3}\left(\mathcal{O}_{B B}, \mathcal{O}_{H}, \mathcal{O}_{6}, \mathcal{O}_{y_{f}}, \mathcal{O}_{L}^{(3) f}, \mathcal{O}_{L}^{f}, \mathcal{O}_{R}^{f}\right)
\end{aligned}
$$

The symbol $\leftrightarrow$ means here that the r.h.s. and l.h.s. can be traded one by the other. We have introduced $\Lambda_{1,2,3}$ which are well-defined linear functions of operators that are common to both basis. For the explicit form of these functions see [23]. 
With that we have shown that the two basis are equivalent in the CP-even sector. Let us finally examine the CP-odd operators. In [27] there 5 such operators, if we exclude the CP-odd triple gluon operators, as we have done. Four of such operators are common: $\mathcal{O}_{B \widetilde{B}}$, $\mathcal{O}_{W \widetilde{W}}, \mathcal{O}_{G \widetilde{G}}, \mathcal{O}_{3 \widetilde{W}}$, see (2.9) and (2.10), but one is different. While they have the operator

$$
\mathcal{O}_{W \widetilde{B}}=g^{\prime} g\left(\Phi^{\dagger} \sigma^{a} \Phi\right) \mathcal{W}_{\mu \nu}^{a} \widetilde{B}^{\mu \nu} .
$$

we have the operator $\mathcal{O}_{H \widetilde{B}}$ in (2.10). Both operators can be related by

$$
\mathcal{O}_{W \widetilde{B}} \leftrightarrow-4 \mathcal{O}_{H \widetilde{B}}-\mathcal{O}_{W \widetilde{W}}
$$

\section{Acknowledgments}

I am thankful to Alex Pomarol for illuminating discussions, and to Joan Elias-Miro, Christophe Grojean and Francesco Riva for a critical reading of the typescript. This work is supported by the CICYT Research Project FPA2011-25948 and by the Generalitat de Catalunya, 2014 SGR 1450.

Open Access. This article is distributed under the terms of the Creative Commons Attribution License (CC-BY 4.0), which permits any use, distribution and reproduction in any medium, provided the original author(s) and source are credited.

\section{References}

[1] CMS collaboration, Search for the standard model Higgs boson decaying into two photons in pp collisions at $\sqrt{s}=7$ TeV, Phys. Lett. B 710 (2012) 403 [arXiv:1202.1487] [INSPIRE].

[2] ATLAS collaboration, Search for the Standard Model Higgs boson in the diphoton decay channel with $4.9 \mathrm{fb}^{-1}$ of pp collisions at $\sqrt{\mathrm{s}}=7 \mathrm{TeV}$ with ATLAS, Phys. Rev. Lett. 108 (2012) 111803 [arXiv:1202.1414] [INSPIRE].

[3] S. Willenbrock and C. Zhang, Effective Field Theory Beyond the Standard Model, arXiv: 1401.0470 [INSPIRE].

[4] F. Bonnet, M.B. Gavela, T. Ota and W. Winter, Anomalous Higgs couplings at the LHC and their theoretical interpretation, Phys. Rev. D 85 (2012) 035016 [arXiv:1105.5140] [InSPIRE].

[5] T. Corbett, O.J.P. Eboli, J. Gonzalez-Fraile and M.C. Gonzalez-Garcia, Constraining anomalous Higgs interactions, Phys. Rev. D 86 (2012) 075013 [arXiv:1207.1344] [InSPIRE].

[6] E. Masso and V. Sanz, Limits on Anomalous Couplings of the Higgs to Electroweak Gauge Bosons from LEP and LHC, Phys. Rev. D 87 (2013) 033001 [arXiv:1211.1320] [InSPIRE].

[7] T. Corbett, O.J.P. Eboli, J. Gonzalez-Fraile and M.C. Gonzalez-Garcia, Robust Determination of the Higgs Couplings: Power to the Data, Phys. Rev. D 87 (2013) 015022 [arXiv: 1211.4580] [INSPIRE].

[8] C. Grojean, E.E. Jenkins, A.V. Manohar and M. Trott, Renormalization Group Scaling of Higgs Operators and $\Gamma(h \rightarrow \gamma \gamma)$, JHEP 04 (2013) 016 [arXiv:1301.2588] [INSPIRE].

[9] J. Elias-Miró, J.R. Espinosa, E. Masso and A. Pomarol, Renormalization of dimension-six operators relevant for the Higgs decays $h \rightarrow \gamma \gamma, \gamma Z$, JHEP 08 (2013) 033 [arXiv:1302.5661] [INSPIRE]. 
[10] W.-F. Chang, W.-P. Pan and F. Xu, Effective gauge-Higgs operators analysis of new physics associated with the Higgs boson, Phys. Rev. D 88 (2013) 033004 [arXiv:1303.7035] [INSPIRE].

[11] B. Dumont, S. Fichet and G. von Gersdorff, A Bayesian view of the Higgs sector with higher dimensional operators, JHEP 07 (2013) 065 [arXiv: 1304.3369] [INSPIRE].

[12] A. Hayreter and G. Valencia, Constraints on anomalous color dipole operators from Higgs boson production at the LHC, Phys. Rev. D 88 (2013) 034033 [arXiv: 1304.6976] [INSPIRE].

[13] S. Banerjee, S. Mukhopadhyay and B. Mukhopadhyaya, Higher dimensional operators and the LHC Higgs data: The role of modified kinematics, Phys. Rev. D 89 (2014) 053010 [arXiv:1308.4860] [INSPIRE].

[14] E. Boos, V. Bunichev, M. Dubinin and Y. Kurihara, Higgs boson signal at complete tree level in the SM extension by dimension-six operators, Phys. Rev. D 89 (2014) 035001 [arXiv: 1309.5410] [INSPIRE].

[15] M. Dahiya, S. Dutta and R. Islam, Unitarizing VV Scattering in Light Higgs Scenarios, arXiv: 1311.4523 [INSPIRE].

[16] J. Elias-Miró, C. Grojean, R.S. Gupta and D. Marzocca, Scaling and tuning of EW and Higgs observables, JHEP 05 (2014) 019 [arXiv:1312.2928] [INSPIRE].

[17] R. Contino, M. Ghezzi, C. Grojean, M. Muhlleitner and M. Spira, eHDECAY: an Implementation of the Higgs Effective Lagrangian into HDECAY, arXiv:1403.3381 [INSPIRE].

[18] J.S. Gainer, J. Lykken, K.T. Matchev, S. Mrenna and M. Park, Beyond Geolocating: Constraining Higher Dimensional Operators in $H \rightarrow 4 \ell$ with Off-Shell Production and More, arXiv: 1403.4951 [INSPIRE].

[19] B. Henning, X. Lu and H. Murayama, What do precision Higgs measurements buy us?, arXiv: 1404.1058 [INSPIRE].

[20] J. Ellis, V. Sanz and T. You, Complete Higgs Sector Constraints on Dimension-6 Operators, JHEP 07 (2014) 036 [arXiv: 1404.3667] [INSPIRE].

[21] G. Amar, S. Banerjee, S. von Buddenbrock, A.S. Cornell, T. Mandal et al., Exploration of the Tensor Structure of the Higgs Boson Coupling to Weak Bosons in $e^{+} e^{-}$Collisions, arXiv: 1405.3957 [INSPIRE].

[22] M. Beneke, D. Boito and Y.-M. Wang, Anomalous Higgs couplings in angular asymmetries of $H \rightarrow Z \ell^{+} \ell^{-}$and $e^{+} e^{-} \rightarrow H Z$, arXiv:1406.1361 [INSPIRE].

[23] J. Elias-Miro, J.R. Espinosa, E. Masso and A. Pomarol, Higgs windows to new physics through $D=6$ operators: constraints and one-loop anomalous dimensions, JHEP 11 (2013) 066 [arXiv:1308.1879] [INSPIRE].

[24] A. Pomarol and F. Riva, Towards the Ultimate SM Fit to Close in on Higgs Physics, JHEP 01 (2014) 151 [arXiv: 1308.2803] [INSPIRE].

[25] R.S. Gupta, A. Pomarol and F. Riva, BSM Primary Effects, arXiv:1405.0181 [INSPIRE].

[26] R. Contino, M. Ghezzi, C. Grojean, M. Muhlleitner and M. Spira, Effective Lagrangian for a light Higgs-like scalar, JHEP 07 (2013) 035 [arXiv: 1303.3876] [INSPIRE].

[27] B. Grzadkowski, M. Iskrzynski, M. Misiak and J. Rosiek, Dimension-Six Terms in the Standard Model Lagrangian, JHEP 10 (2010) 085 [arXiv: 1008.4884] [INSPIRE]. 
[28] G.F. Giudice, C. Grojean, A. Pomarol and R. Rattazzi, The Strongly-Interacting Light Higgs, JHEP 06 (2007) 045 [hep-ph/0703164] [INSPIRE].

[29] M.E. Peskin and T. Takeuchi, A New constraint on a strongly interacting Higgs sector, Phys. Rev. Lett. 65 (1990) 964 [INSPIRE].

[30] K. Hagiwara, R.D. Peccei, D. Zeppenfeld and K. Hikasa, Probing the Weak Boson Sector in $e^{+} e^{-} \rightarrow W^{+} W^{-}$, Nucl. Phys. B 282 (1987) 253 [InSPIRE].

[31] K. Hagiwara, S. Ishihara, R. Szalapski and D. Zeppenfeld, Low-energy effects of new interactions in the electroweak boson sector, Phys. Rev. D 48 (1993) 2182 [INSPIRE].

[32] A. De Rújula, M.B. Gavela, P. Hernández and E. Masso, The Selfcouplings of vector bosons: Does LEP-1 obviate LEP-2?, Nucl. Phys. B 384 (1992) 3 [INSPIRE].

[33] A. Hoecker, The Hadronic Contribution to the Muon Anomalous Magnetic Moment and to the Running Electromagnetic Fine Structure Constant at $M Z$ - Overview and Latest Results, Nucl. Phys. Proc. Suppl. 218 (2011) 189 [arXiv:1012.0055] [INSPIRE]. 